\title{
Input and Output Performance of M-MRAC in the Presence of Bounded Disturbances
}

\author{
Vahram Stepanyan* \\ Mission Critical Technologies Inc, NASA Ames Research Center, Moffett Field, CA 94035, \\ Kalmanje Krishnakumar ${ }^{\dagger}$ \\ NASA Ames Research Center, Moffett Field, CA 94035
}

\begin{abstract}
This paper presents performance analysis of novel modified model reference adaptive control (M-MRAC) architecture in conjunction with different robust adaptive laws for the uncertain linear systems subject to unknown but bounded disturbances. It is shown that for any robust adaptive law the tracking error of the M-MRAC system can be arbitrarily decreased in transient and in the steady-state by increasing the adaptation rate, without generating high frequency oscillations in the control signal, which are unavoidable in conventional MRAC systems for large adaptation rates. Moreover, the generated adaptive control signal arbitrary closely tracks the ideal control signal when the error feedback gain is simultaneously increased with the adaptation rate, according to derived rule. The results are demonstrated via simulations.
\end{abstract}

\section{Introduction}

The transient performance analysis of model reference adaptive control (MRAC) systems has been a challenging research field since the stabilizing control algorithms had been developed. The reason is the dependence of the performance bounds on the unknown quantities, which makes it difficult to quantify them a priori and to develop algorithms to systematically improve them. Even though asymptotic tracking can be achieved in many systems, the transient performance of the adaptive controllers can be quite poor. ${ }^{21}$ There have been great efforts to modify the control architecture and the adaptive laws from the perspective of improving the transient behavior of the output tracking error. The majority of these efforts led to high gain linear nonadaptive feedbacks, ${ }^{3,4,20,22}$ switching control laws, ${ }^{10,11}$ or to a parameter dependent persistent excitation conditions. ${ }^{1}$

Meantime, the behavior of the adaptive control signal has got less attention. First contribution to transient analysis of the the adaptive control signal can be found in,$^{5}$ where it is shown that the bound on the control signal is proportional to the square root of the adaptation rate. This result is conservative, but it reflects the general observations about the control signal behavior of the MRAC system. Namely, increasing the adaptation rate, while reducing the tracking error magnitude (see for example ${ }^{9}$ ), generates high frequency oscillations and big overshoot in the control signal leading to possible actuator failures or excitation of unmodeled dynamics, which in turn can drive the overall system to instability. This shortcoming is common for the majority of existing adaptive control methods. Recently some results have been appearing in the control community, which guarantee improved transient behavior of the control signal with the increase of the adaptation rate. These results are mainly based on the $L_{1}$ design method, introduced in the Ref. ${ }^{2}$ An alternative approach is proposed in, ${ }^{19}$ which along with the tracking error, also uses its integral to guarantee the transient performance of both input and output signals.

In, ${ }^{18}$ we introduce the consent of modified reference model MRAC (M-MRAC) architecture, which was extended to to multi-input multi-output linear systems in. ${ }^{17}$ This approach is motivated by the fact that the initial large error in the control gains generates large transient excursions both in system's control and output signals. Therefore, driving the reference model toward the system proportional to the tracking error

\footnotetext{
*Senior Scientist, Mission Critical Technologies Inc., Senior Member AIAA, vahram.stepanyan@nasa.gov

${ }^{\dagger}$ Principal Investigator, Integrated Resilient Aircraft Control Project, Intelligent Systems Division, Associate Fellow AIAA, kalmanje.krishnakumar@nasa.gov
} 
prevents the system's attempt to aggressively maneuver toward the reference model. As the tracking error approaches zero, the reference model approaches its original form, which is called an ideal reference model to distinguish between the modified and unmodified reference models. It has been shown in ${ }^{18}$ that the error feedback term determines the damping in the control signal, increasing of which makes it possible to increase the learning rate for better transient performance without generating high frequency oscillations in the adaptive system. A design guideline has been provided for the selection of the feedback gain relative to the adaptation rate.

In the presence of bounded disturbances, the transient performance analysis of input and output signals are more involved, since the adaptive algorithms need to be modified in order to prevent the parameter drift. Some modifications include the projection projection based adaptive law, ${ }^{15} e$-modification, ${ }^{12} \sigma$-modification ${ }^{7}$ or the dead-zone technique. ${ }^{14,16}$ In this paper we investigate the performance of M-MRAC systems in the presence of bounded disturbances. We show that the tracking error and the control error can be decreased as desired by increasing the adaptation rate and the error feedback gain. These transient properties of the M-MRAC systems are uniform for all type of robust modifications of the adaptive laws, which guarantee a priori boundedness of the parameter estimates. It is also shown that the M-MRAC algorithm enables the designer to prevent the bursting phenomena, which is known to occur in the adaptive systems when the external input is not sufficiently rich (see for example Ref. ${ }^{6}$ ).

The rest of the paper is organized as follows. In Section II we state the problem and present the control design. The error signals are defined in Section III. In Section IV we analyze performance of M-MRAC with $\sigma$-modification adaptive laws. Sections V and VI discuss the differences with other robust modifications. Section VII presents analysis of the bursting phenomena and some concluding comments are given in Section VIII.

\section{Problem formulation}

Consider a multi-input multi-output uncertain linear system

$$
\dot{\boldsymbol{x}}(t)=A \boldsymbol{x}(t)+B[\boldsymbol{u}(t)-\boldsymbol{f}(t)], \quad \boldsymbol{x}(0)=\boldsymbol{x}_{0},
$$

where $\boldsymbol{x} \in \mathbb{R}^{n}$ and $\boldsymbol{u} \in \mathbb{R}^{q}$ are the state and input of the system, $\boldsymbol{f}(t) \in \mathbb{R}^{q}$ is bounded and piece-wise continuous external disturbance, and $A \in \mathbb{R}^{n \times n}$ and $B \in \mathbb{R}^{n \times q}$ are unknown constant matrices satisfying the following matching conditions.

Assumption II.1 Given a Hurwitz matrix $A_{m} \in \mathbb{R}^{n \times n}$ and a matrix $B_{m} \in \mathbb{R}^{n \times q}$ of full column rank, there exists a matrix $K_{1} \in \mathbb{R}^{q \times n}$ and a sign definite matrix $\Lambda \in \mathbb{R}^{q \times q}$ such that the following equations hold

$$
\begin{aligned}
B & =B_{m} \Lambda \\
A & =A_{m}-B K_{1} .
\end{aligned}
$$

Remark II.1 The sign definiteness of $\Lambda$ corresponds to the conventional sign condition on the high frequency gain matrix of MIMO systems (see for example Ref. ${ }^{13}$ ). Without loss of generality we assume that $\Lambda$ is positive definite. The rest of of the conditions for the existence of an adaptive controller are given by the equations (2).

The control objective is to design a control signal $\boldsymbol{u}(t)$ such that the system tracks the reference model

$$
\dot{\boldsymbol{x}}^{0}(t)=A_{m} x^{0}(t)+B_{m} N \boldsymbol{r}(t), \quad \boldsymbol{x}^{0}(0)=\boldsymbol{x}_{0}
$$

where $A_{m}, B_{m}$ are chosen according to performance specifications and satisfy Assumption II.1, and $\boldsymbol{r}(t)$ is a bounded and smooth external command. The matrix $N=-\left(C A_{m}^{-1} B_{m}\right)^{-1}$ is chosen such that the output $\boldsymbol{y}^{0}(t)=C \boldsymbol{x}^{0}(t)$ perfectly tracks a constant external command. To achieve this objective we use the novel M-MRAC architecture introduced in Ref., ${ }^{18}$ where we refer to the system (3) as the ideal reference model.

We notice that the system (1) can be written in the form

$$
\dot{\boldsymbol{x}}(t)=A_{m} \boldsymbol{x}(t)+B_{m} N \boldsymbol{r}(t)+B_{m} \Lambda\left[\boldsymbol{u}(t)-K_{1} \boldsymbol{x}(t)-K_{2} \boldsymbol{r}(t)-\boldsymbol{f}(t)\right],
$$

where $K_{2}=\Lambda^{-1} N$. Hence the application of the ideal control signal

$$
\boldsymbol{u}^{0}(t)=K_{1} \boldsymbol{x}^{0}(t)+K_{2} \boldsymbol{r}(t)+\boldsymbol{f}(t),
$$


translats the system into the ideal reference model (3). The ideal reference model (3) always can be specified from the performance perspectives, but the ideal control signal (5) cannot be implemented since the matrices $K_{1}, K_{2}$ and the vector-function $\boldsymbol{f}(t)$ are assumed to be unknown.

We design the adaptive control according to M-MRAC architecture as

$$
\boldsymbol{u}(t)=\hat{K}_{1}(t) \boldsymbol{x}(t)+\hat{K}_{2}(t) \boldsymbol{r}(t)+\hat{\boldsymbol{f}}(t),
$$

where $\hat{K}_{1}(t)$ and $\hat{K}_{2}(t)$ are the estimates of the ideal control gains $K_{1}$ and $K_{2}$, and $\hat{\boldsymbol{f}}(t)$ is the estimate of a constant vector $\overline{\boldsymbol{f}}$ that can be referred to as an "average" value of $\boldsymbol{f}(t)$. These estimates are updated online according to robust adaptive laws

$$
\begin{aligned}
\dot{\hat{K}}_{1}(t) & =-\gamma B_{m}^{\top} P \boldsymbol{e}(t) \boldsymbol{x}^{\top}(t)+\Psi_{1}\left(\boldsymbol{x}(t), \boldsymbol{e}(t), \hat{K}_{1}(t)\right) \\
\dot{\hat{K}}_{2}(t) & =-\gamma B_{m}^{\top} P \boldsymbol{e}(t) \boldsymbol{r}^{\top}(t)+\Psi_{2}\left(\boldsymbol{r}(t), \boldsymbol{e}(t), \hat{K}_{2}(t)\right) \\
\dot{\hat{\boldsymbol{f}}}(t) & =-\gamma B_{m}^{\top} P \boldsymbol{e}(t)+\Psi_{3}(\boldsymbol{e}(t), \hat{\boldsymbol{f}}(t)),
\end{aligned}
$$

where $\gamma>0$ is the adaptation rate, $P=P^{\top}>0$ is the solution of the Lyapunov equation

$$
A_{m}^{\top} P+P A_{m}=-Q
$$

for some $Q=Q^{\top}>0$, and the terms $\Psi_{1}, \Psi_{2}, \Psi_{3}$ represent the robust modifications such as $\sigma$-modification, $e$-modification, projection operator or dead-zone modification. Here $\boldsymbol{e}(t)=\boldsymbol{x}(t)-\boldsymbol{x}_{m}(t)$ is the tracking error between the system and the modified reference model

$$
\dot{\boldsymbol{x}}_{m}(t)=A_{m} x_{m}(t)+B_{m} N \boldsymbol{r}(t)+\lambda\left[\boldsymbol{x}(t)-\boldsymbol{x}_{m}(t)\right], \quad \boldsymbol{x}_{m}(0)=\boldsymbol{x}_{0},
$$

where $\lambda>0$ is a design parameter that specifies the tracking error feedback into the reference model.

\section{Error signals}

Introducing the parameter estimation errors as $\tilde{K}_{1}(t)=\hat{K}_{1}(t)-K_{1}$ and $\tilde{K}_{2}(t)=\hat{K}_{2}(t)-K_{2}$, the dynamics of the tracking error $\boldsymbol{e}(t)$ can be written in the form

$$
\dot{\boldsymbol{e}}(t)=\left(A_{m}-\lambda \mathbb{I}_{n}\right) \boldsymbol{e}(t)+B_{m} \Lambda\left[\tilde{K}_{1}(t) \boldsymbol{x}(t)+\tilde{K}_{2}(t) \boldsymbol{r}(t)+\hat{\boldsymbol{f}}(t)-\boldsymbol{f}(t)\right],
$$

where $\mathbb{I}_{n}$ denotes $n$-dimensional identity matrix. Since the control objective is to track the state of the ideal reference model $\boldsymbol{x}^{0}(t)$, for the analysis we also need the error signal $\boldsymbol{e}^{0}(t)=\boldsymbol{x}(t)-\boldsymbol{x}^{0}(t)$, the dynamics of which is given by the equation

$$
\dot{\boldsymbol{e}}^{0}(t)=A_{m} \boldsymbol{e}^{0}(t)+B_{m} \Lambda\left[\tilde{K}_{1}(t) \boldsymbol{x}(t)+\tilde{K}_{2}(t) \boldsymbol{r}(t)+\hat{\boldsymbol{f}}(t)-\boldsymbol{f}(t)\right] .
$$

Therefore, it is straightforward to relate the tracking errors $\boldsymbol{e}(t)$ and $\boldsymbol{e}^{0}(t)$

$$
\frac{d}{d t}\left[e(t)-e^{0}(t)\right]=A_{m}\left[e(t)-e^{0}(t)\right]-\lambda e(t),
$$

Since $A_{m}$ is Hurwitz, it follows from the equation (12) that $\boldsymbol{e}^{0}(t)$ is bounded, if $\boldsymbol{e}(t)$ is bounded. Moreover, let $\boldsymbol{e}(t) \in \mathcal{L}_{\infty}$ and $\Phi(t-\tau)=\exp \left(A_{m}(t-\tau)\right)$ be the state transition matrix corresponding to $A_{m}$. Since, there exists a positive constants $k_{m}$ such that $\|\Phi(t-\tau)\|_{\mathcal{L}_{1}} \leq k_{m}$ for all $\tau \geq 0$, $t \geq \tau$, it follows from Ref. ${ }^{8}$ (p. 200) that the following inequality holds

$$
\left\|\boldsymbol{e}^{0}(t)-\boldsymbol{e}(t)\right\|_{\mathcal{L}_{\infty}} \leq \lambda\|\Phi(t-\tau)\|_{\mathcal{L}_{1}}\|\boldsymbol{e}(t)\|_{\mathcal{L}_{\infty}}
$$

The specific value of $k_{m}$ is not of our interest, because the matrix $A_{m}$ is fixed from the performance perspectives, and the dependence of the error signals behavior on the choice of $A_{m}$ is not considered here. The norm bound on the error signal $e^{0}(t)$ is readily obtained from (13) in the form

$$
\left\|\boldsymbol{e}^{0}(t)\right\|_{\mathcal{L}_{\infty}} \leq\left(1+\lambda k_{m}\right)\|\boldsymbol{e}(t)\|_{\mathcal{L}_{\infty}} .
$$


In the following analysis we will also need the control error signal that is defined as $\tilde{\boldsymbol{u}}(t)=\boldsymbol{u}(t)-\boldsymbol{u}^{0}(t)$. From M-MRAC architecture it follows that

$$
\begin{aligned}
\tilde{\boldsymbol{u}}(t) & =\hat{K}_{1}(t) \boldsymbol{x}(t)+\hat{K}_{2}(t) \boldsymbol{r}(t)-K_{1}(t) \boldsymbol{x}^{0}(t)-K_{2} \boldsymbol{r}(t)+\hat{\boldsymbol{f}}(t)-\boldsymbol{f}(t) \\
& =\tilde{K}_{1}(t) \boldsymbol{x}(t)+\tilde{K}_{2} \boldsymbol{r}(t)+\hat{\boldsymbol{f}}(t)-\boldsymbol{f}(t)+K_{1} \boldsymbol{e}^{0}(t) .
\end{aligned}
$$

Therefore, the error dynamics (10) can be also represented in the form

$$
\dot{\boldsymbol{e}}(t)=\left(A_{m}-\lambda \mathbb{I}_{n}\right) \boldsymbol{e}(t)+B_{m} \Lambda\left[\tilde{\boldsymbol{u}}(t)-K_{1}^{\top} \boldsymbol{e}^{0}(t)\right],
$$

Since the ideal control signal is the best achievable signal, we are interested in minimizing the control error $\tilde{\boldsymbol{u}}(t)$, as well as the tracking error signals $\boldsymbol{e}(t)$ and $\boldsymbol{e}^{0}(t)$, both in transient and steady state by selecting proper values for the adaptation rate $\gamma$ and feedback parameter $\lambda$. This is the main objective of the analysis in the following sections, which we provide for different robust modification terms.

Remark III.1 When $\lambda=0$ the proposed control architecture is identical with the conventional MRAC, that is the only difference from the MRAC design is the modification of the reference model by the term $\lambda \boldsymbol{e}(t)$.

\section{Performance analysis of M-MRAC with $\sigma$-modification}

In this section, we provide asymptotic and transient analysis of the M-MRAC architecture with the $\sigma$-modification adaptive laws, that is we set

$$
\begin{aligned}
\Psi_{1}\left(\boldsymbol{x}, \boldsymbol{e}, \hat{K}_{1}\right) & =-\sigma \hat{K}_{1} \\
\Psi_{2}\left(\boldsymbol{r}, \boldsymbol{e}, \hat{K}_{2}\right) & =-\sigma \hat{K}_{2} \\
\Psi_{3}(\boldsymbol{e}, \hat{\boldsymbol{f}}) & =-\sigma \hat{\boldsymbol{f}},
\end{aligned}
$$

where $\sigma>0$ is a design parameter.

\section{A. Stability}

The following theorem gives the stability properties of M-MRAC architecture with $\sigma$-modification for the uncertain systems subject to bounded disturbances.

Theorem IV.1 Let the system (1) be controlled by the M-MRAC scheme given by (6), (9) and (7) with (17). Then all closed-loop signals are bounded.

Proof. Consider the following candidate Lyapunov function

$$
V(t)=\boldsymbol{e}^{\top}(t) P \boldsymbol{e}(t)+\frac{1}{\gamma} \operatorname{tr}\left(\tilde{K}_{1}^{\top}(t) \tilde{K}_{1}(t) \Lambda+\tilde{K}_{2}^{\top}(t) \tilde{K}_{2}(t) \Lambda+\tilde{\boldsymbol{f}}^{\top}(t) \tilde{\boldsymbol{f}}(t) \Lambda\right)
$$

where $\tilde{\boldsymbol{f}}(t)=\hat{\boldsymbol{f}}(t)-\overline{\boldsymbol{f}}$. Its derivative computed along the trajectories of the systems (10) and (7) with (17) takes the form

$$
\begin{aligned}
\dot{V}(t) & =-e^{\top}(t) Q \boldsymbol{e}(t)-2 \lambda e^{\top}(t) P \boldsymbol{e}(t)+2 e^{\top}(t) P B_{m} \Lambda(\overline{\boldsymbol{f}}-\boldsymbol{f}(t)) \\
& -2 \frac{\sigma}{\gamma} \operatorname{tr}\left(\tilde{K}_{1}^{\top}(t) \hat{K}_{1}(t) \Lambda+\tilde{K}_{2}^{\top}(t) \hat{K}_{2}(t) \Lambda+\tilde{\boldsymbol{f}}^{\top}(t) \hat{\boldsymbol{f}}(t) \Lambda\right) .
\end{aligned}
$$

Completing the squares in (19) we obtain

$$
\dot{V}(t) \leq-a_{1}\|e(t)\|^{2}-\frac{\sigma}{\gamma} \lambda_{\min }(\Lambda)\left(\left\|\tilde{K}_{1}(t)\right\|_{F}^{2}+\left\|\tilde{K}_{2}(t)\right\|_{F}^{2}+\|\tilde{\boldsymbol{f}}(t)\|^{2}\right)+c
$$

where $a_{1}=\lambda_{\min }(Q)+2 \lambda_{\min }(P) \lambda-1, \lambda_{\min }(Q)$ denotes the minimum eigenvalue of the matrix $Q$, $\|\boldsymbol{e}\|$ denotes the Euclidean norm of the vector $\boldsymbol{e}, f_{*}=\left\|P B_{m} \Lambda(\overline{\boldsymbol{f}}-\boldsymbol{f}(t))\right\|_{\mathcal{L}_{\infty}}, k_{1}=\left\|K_{1}\right\|_{F}$ and $k_{2}=\left\|K_{2}\right\|_{F}$, and the subscript $F$ denotes the Frobenius matrix norm, $f_{0}=\|\overline{\boldsymbol{f}}\|$ and $c=f_{*}^{2}+\frac{\sigma}{\gamma} \lambda_{\max }(\Lambda)\left(k_{1}^{2}+k_{2}^{2}+f_{0}^{2}\right)$. Choosing $Q$ such that $\lambda_{\min }(Q)-1 \geq 0$, we conclude that $\dot{V}(t)$ is negative semi-definite outside the compact set

$$
\Omega=\left\{\left(\boldsymbol{e}, \tilde{K}_{1}, \tilde{K}_{2}, \tilde{\boldsymbol{f}}\right): a_{1}\|e\|^{2}+\frac{\sigma}{\gamma} \lambda_{\min }(\Lambda)\left(\left\|\tilde{K}_{1}\right\|_{F}^{2}+\left\|\tilde{K}_{2}\right\|_{F}^{2}+\|\tilde{\boldsymbol{f}}\|^{2}\right) \leq c\right\} .
$$


Therefore the signals $\boldsymbol{e}(t), \tilde{K}_{1}(t), \tilde{K}_{2}(t), \tilde{\boldsymbol{f}}(t)$ are uniformly ultimately bounded. The boundedness of $\boldsymbol{e}^{0}(t)$ follows from the inequality (13). Also, the boundedness of error signals implies the boundedness of $\boldsymbol{x}(t)$, $\hat{K}_{1}(t), \hat{K}_{2}(t)$ and $\hat{\boldsymbol{f}}(t)$. Hence $\boldsymbol{u}(t)$ and $\tilde{\boldsymbol{u}}(t)$ are bounded as well.

\section{B. Transient behavior of the tracking error}

Theorem IV.1 guarantees the boundedness of all closed-loop signals in the system (1), (9) and (7) with (17). Therefore, there exist constant $k_{1}^{*}>0, k_{2}^{*}>0$ such that

$$
\begin{aligned}
& \operatorname{tr}\left(\tilde{K}_{1}^{\top}(t) \hat{K}_{1}(t) \Lambda+\tilde{K}_{2}^{\top}(t) \hat{K}_{2}(t) \Lambda+\tilde{\boldsymbol{f}}^{\top}(t) \hat{\boldsymbol{f}}(t) \Lambda\right) \leq k_{1}^{*} \\
& \operatorname{tr}\left(\tilde{K}_{1}^{\top}(t) \tilde{K}_{1}(t) \Lambda+\tilde{K}_{2}^{\top}(t) \tilde{K}_{2}(t) \Lambda+\tilde{\boldsymbol{f}}^{\top}(t) \tilde{\boldsymbol{f}}(t) \Lambda\right) \leq k_{2}^{*} .
\end{aligned}
$$

Then, it follows from (19) that $\dot{V}(t) \leq 0$ if

$$
\|\boldsymbol{e}(t)\| \geq \frac{f_{*}}{a_{2}}+\sqrt{\left[\frac{f_{*}}{a_{2}}\right]^{2}+\frac{2 \sigma}{a_{2} \gamma} k_{1}^{*}} .
$$

where we denote $a_{2}=\lambda_{\min }(Q)+2 \lambda_{\min }(P) \lambda$. To obtain a bound on $\|\boldsymbol{e}(t)\|$ we consider a Lyapunov level set defined as

$$
L=\left\{\left(\boldsymbol{e}, \tilde{K}_{1}, \tilde{K}_{2}, \tilde{\boldsymbol{f}}\right): V\left(\boldsymbol{e}, \tilde{K}_{1}, \tilde{K}_{2}, \tilde{\boldsymbol{d}}\right)=V^{*}\right\}
$$

with

$$
V^{*} \triangleq \lambda_{\max }(P)\left(\frac{f_{*}}{a_{2}}+\sqrt{\left[\frac{f_{*}}{a_{2}}\right]^{2}+\frac{2 \sigma}{a_{2} \gamma} k_{1}^{*}}\right)^{2}+\frac{1}{\gamma} k_{2}^{*} .
$$

It is easy to see that $\dot{V}(t) \leq 0$ whenever $V(t) \geq V^{*}$. Indeed, from the definition of $V(t)$ it follows that

$$
\lambda_{\max }(P)\|\boldsymbol{e}(t)\|^{2}+\frac{1}{\gamma} k_{2}^{*} \geq V(t) \geq V^{*},
$$

which implies that $\|\boldsymbol{e}(t)\|$ satisfies the inequality (23). Therefore, $V(t) \leq V^{*}$ for all $t$. On the other hand, from the definition of $V(t)$ we have

$$
\lambda_{\min }(P)\|\boldsymbol{e}(t)\|^{2} \leq \boldsymbol{e}^{\top}(t) P \boldsymbol{e}(t) \leq V(t) .
$$

Therefore

$$
\|\boldsymbol{e}(t)\| \leq \sqrt{\frac{\lambda_{\max }(P)}{\lambda_{\min }(P)}\left[\frac{f_{*}}{a_{2}}+\sqrt{\left[\frac{f_{*}}{a_{2}}\right]^{2}+\frac{2 \sigma}{a_{2} \gamma} k_{1}^{*}}\right]^{2}+\frac{1}{\gamma \lambda_{\min }(P)} k_{2}^{*}} .
$$

Since the inequality (27) holds uniformly in $t,\|\boldsymbol{e}(t)\|_{\mathcal{L}_{\infty}}$ satisfies the same bound. The bound on the actual tracking error $\left\|\boldsymbol{e}^{0}(t)\right\|_{\mathcal{L}_{\infty}}$ follows from the inequality (13) and has the form

$$
\left\|\boldsymbol{e}^{0}(t)\right\|_{\mathcal{L}_{\infty}} \leq\left(1+\lambda k_{m}\right) \sqrt{\frac{\lambda_{\max }(P)}{\lambda_{\min }(P)}\left[\frac{f_{*}}{a_{2}}+\sqrt{\left[\frac{f_{*}}{a_{2}}\right]^{2}+\frac{2 \sigma}{a_{2} \gamma} k_{1}^{*}}\right]^{2}+\frac{1}{\gamma \lambda_{\min }(P)} k_{2}^{*}}
$$

We notice that the derived bound on $\left\|e^{0}(t)\right\|_{\mathcal{L}_{\infty}}$ cannot be arbitrarily decreased by increasing the design parameters $\lambda$ and $\gamma$. If we set $\lambda=c_{0} \sqrt{\gamma}$, the following asymptotic bound can be written

$$
\lim _{\gamma \rightarrow \infty}\left\|\boldsymbol{e}^{0}(t)\right\|_{\mathcal{L}_{\infty}} \leq k_{m} \sqrt{\frac{\lambda_{\max }(P)}{\lambda_{\min }^{3}(P)} f_{*}^{2}+\frac{c_{0}}{\lambda_{\min }(P)} k_{2}^{*}},
$$


which still can be reduced by increasing $\lambda_{\min }(P)$.

For the MRAC design, that is when $\lambda=0$, the bounds (28) and (29) are replaced with

$$
\begin{aligned}
\left\|\boldsymbol{e}^{0}(t)\right\|_{\mathcal{L}_{\infty}} & \leq \sqrt{\frac{\lambda_{\max }(P)}{\lambda_{\min }(P)}\left[\frac{f_{*}}{\lambda_{\min }(Q)}+\sqrt{\left[\frac{f_{*}}{\lambda_{\min }(Q)}\right]^{2}+\frac{2 \sigma}{\lambda_{\min }(Q) \gamma} k_{1}^{*}}\right]^{2}+\frac{1}{\gamma \lambda_{\min }(P)} k_{2}^{*}} \\
\lim _{\gamma \rightarrow \infty}\left\|\boldsymbol{e}^{0}(t)\right\|_{\mathcal{L}_{\infty}} & \leq \frac{f_{*}}{\lambda_{\min }(Q)} \sqrt{2 \frac{\lambda_{\max }(P)}{\lambda_{\min }(P)}}
\end{aligned}
$$

respectively. For small values of the design parameters $\gamma$ and $\lambda$, the bounds (28) and (31) are comparable. However, when both parameters are large, the asymptotic bounds (29) and (31) differ by the term $\frac{c_{0}}{\lambda_{\min }(P)} k_{2}^{*}$ in the square root. This difference vanishes, when $\lambda_{\min }(Q)$ takes large values. Therefore, the transient behavior of the tracking error in both MRAC and M-MRAC designs can be improved as desired, if the design parameters $\lambda, \gamma$ and $Q$ can be chosen arbitrary large. As we show in the next subsection, fast adaptation also improves the transient behavior of the control signal in M-MRAC design, while generating high frequency oscillations in the control signal of the MRAC design.

When the function $\boldsymbol{f}(t)$ has a bounded derivative, tighter bounds can be obtained. Let $\left\|\boldsymbol{f}(t)+\frac{1}{\sigma} \dot{\boldsymbol{f}}(t)\right\|_{\mathcal{L}_{\infty}} \leq$ $f_{1}$ for some positive constant $f_{1}$ and $\tilde{\boldsymbol{f}}(t)=\hat{\boldsymbol{f}}(t)-\boldsymbol{f}(t)$. Then, the derivative of the candidate Lyapunov function $V(t)$ in (18) is computed as follows

$$
\begin{aligned}
\dot{V}(t) & =-e^{\top}(t) Q \boldsymbol{e}(t)-2 \lambda e^{\top}(t) P \boldsymbol{e}(t) \\
& -2 \frac{\sigma}{\gamma} \operatorname{tr}\left(\left[\tilde{K}_{1}^{\top}(t) \hat{K}_{1}(t)+\tilde{K}_{2}^{\top}(t) \hat{K}_{2}(t)+\tilde{\boldsymbol{f}}^{\top}(t) \hat{\boldsymbol{f}}(t)+\frac{1}{\sigma} \tilde{\boldsymbol{f}}^{\top}(t) \dot{\boldsymbol{f}}(t)\right] \Lambda\right) .
\end{aligned}
$$

Completing the squares in (32) results in

$$
\dot{V}(t) \leq-a_{2}\|\boldsymbol{e}(t)\|^{2}-\frac{\sigma}{\gamma} \lambda_{\min }(\Lambda)\left(\left\|\tilde{K}_{1}(t)\right\|_{F}^{2}+\left\|\tilde{K}_{2}(t)\right\|_{F}^{2}+\|\tilde{\boldsymbol{f}}(t)\|^{2}\right)+\frac{\sigma}{\gamma} c_{1},
$$

where we denote $c_{1}=\lambda_{\max }(\Lambda)\left(k_{1}^{2}+k_{2}^{2}+f_{1}^{2}\right)$. Obviously, $\dot{V}(t) \leq 0$ outside the compact set

$$
\Omega_{1}=\left\{\left(\boldsymbol{e}, \tilde{K}_{1}, \tilde{K}_{2}, \tilde{\boldsymbol{f}}\right): a_{2}\|\boldsymbol{e}\|^{2}+\frac{\sigma}{\gamma} \lambda_{\min }(\Lambda)\left(\left\|\tilde{K}_{1}\right\|_{F}^{2}+\left\|\tilde{K}_{2}\right\|_{F}^{2}+\|\tilde{\boldsymbol{f}}\|^{2}\right) \leq \frac{\sigma}{\gamma} c_{1}\right\} .
$$

As in the previous subsection, we can conclude from the inequality (33) that the signals $\boldsymbol{e}(t), \tilde{K}_{1}(t), \tilde{K}_{2}(t), \tilde{\boldsymbol{f}}(t)$ are uniformly ultimately bounded, which implies that the signals $\boldsymbol{e}^{0}(t), \boldsymbol{x}(t), \hat{K}_{1}(t), \hat{K}_{2}(t), \hat{\boldsymbol{f}}(t), \boldsymbol{u}(t)$ and $\tilde{\boldsymbol{u}}(t)$ are bounded as well. In fact, the trajectories stay inside the Lyapunov level set

$$
L_{1}=\left\{\left(\boldsymbol{e}, \tilde{K}_{1}, \tilde{K}_{2}, \tilde{\boldsymbol{f}}\right): V\left(\boldsymbol{e}, \tilde{K}_{1}, \tilde{K}_{2}, \tilde{\boldsymbol{d}}\right)=\lambda_{\max }(P) \frac{\sigma}{a_{2} \gamma} c_{1}+\frac{1}{\gamma} k_{2}^{*}\right\},
$$

where $k_{2}^{*}$ is defined in (22). Therefore, the following conservative bound can be derived

$$
\|\boldsymbol{e}(t)\|_{\mathcal{L}_{\infty}} \leq \sqrt{\frac{\lambda_{\max }(P)}{\lambda_{\min }(P)} \frac{\sigma}{a_{2} \gamma} c_{1}+\frac{1}{\gamma \lambda_{\min }(P)} k_{2}^{*}} .
$$

It follows from the inequality (34) that $\|\boldsymbol{e}(t)\|_{\mathcal{L}_{\infty}}$ can be decreased as desired by increasing $\gamma$. On the other hand the actual tracking error satisfies the norm bound

$$
\left\|\boldsymbol{e}^{0}(t)\right\|_{\mathcal{L}_{\infty}} \leq\left(1+k_{m} \lambda\right) \sqrt{\frac{\lambda_{\max }(P)}{\lambda_{\min }(P)} \frac{\sigma}{a_{2} \gamma} c_{1}+\frac{1}{\gamma \lambda_{\min }(P)} k_{2}^{*}},
$$

which results in the limiting equality

$$
\lim _{\gamma \rightarrow \infty}\left\|\boldsymbol{e}^{0}(t)\right\|_{\mathcal{L}_{\infty}} \leq k_{m} \sqrt{\frac{c_{0}}{\lambda_{\min }(P)} k_{2}^{*}},
$$

when we set $\lambda=c_{0} \sqrt{\gamma}$. Clearly, this asymptotic bound can be further decrease by increasing $\lambda_{\min }(P)$. The transient behavior of tracking error signal is given by the following theorem. 
Theorem IV.2 Let the system (1) be controlled by the M-MRAC scheme given by (6), (9) and (7) with (57), and $\lambda$ is selected as $\lambda=c_{0} \sqrt{\gamma}$ for some positive constant $c_{0}$. Then for large $\gamma$, the $\mathcal{L}_{\infty}$ norm of the tracking error $e^{0}(t)$ can be bounded as follows

$$
\left\|\boldsymbol{e}^{0}(t)\right\|_{\mathcal{L}_{\infty}} \leq O\left(k_{m} \sqrt{\frac{\lambda_{\max }(P)}{\lambda_{\min }^{3}(P)} f_{*}^{2}+\frac{c_{0}}{\lambda_{\min }(P)} k_{2}^{*}}\right) .
$$

Moreover, if the disturbance term has a bounded derivative, then

$$
\left\|\boldsymbol{e}^{0}(t)\right\|_{\mathcal{L}_{\infty}} \leq O\left(k_{m} \sqrt{\frac{c_{0}}{\lambda_{\min }(P)} k_{2}^{*}}\right),
$$

\section{Transient behavior of the control signal}

Now we derive a bound on $\|\tilde{\boldsymbol{u}}(t)\|_{\mathcal{L}_{\infty}}$. To this end, we assume that $\boldsymbol{f}(t)$ has bounded time derivatives. This assumption is only needed for the analysis purposes and is conditioned on the way the bound on the control error is derived. The final result is independent of this assumption and involves only the bound on the disturbance $\boldsymbol{f}(t)$. To this end we recall that $\tilde{\boldsymbol{u}}(t)$ does not explicitly depend on design parameters $\lambda$ and $\gamma$. Instead, $\dot{\tilde{\boldsymbol{u}}}(t)$ depend on $\gamma$ through the adaptive laws, and $\ddot{\tilde{\boldsymbol{u}}}(t)$ depend on $\lambda$ through the tracking error dynamics. Differentiating $\tilde{\boldsymbol{u}}(t)$ and substituting the adaptive laws we obtain

$$
\dot{\tilde{\boldsymbol{u}}}(t)=-\gamma \rho(t) B_{m}^{\top} P \boldsymbol{e}(t)-\sigma\left[\tilde{\boldsymbol{u}}(t)+\boldsymbol{u}^{0}(t)\right]+\boldsymbol{r}_{a}(t)-\dot{\boldsymbol{f}}(t),
$$

where we denote

$$
\begin{aligned}
\rho(t) & =\boldsymbol{x}^{\top}(t) \boldsymbol{x}(t)+\boldsymbol{r}^{\top}(t) \boldsymbol{r}(t)+1 \\
\boldsymbol{r}_{a}(t) & =\tilde{K}_{1}(t) \dot{\boldsymbol{x}}(t)+\tilde{K}_{2}(t) \dot{\boldsymbol{r}}(t)+K_{1} \dot{\boldsymbol{e}}^{0}(t),
\end{aligned}
$$

From the results of the previous section it follows that all signals involved in the equation (39) are bounded. In particular, there exist positive constants $\alpha_{1}, \alpha_{2}, \alpha_{3}$ such that $\|\rho(t)\|_{\mathcal{L}_{\infty}} \leq \alpha_{1},\|\dot{\rho}(t)\|_{\mathcal{L}_{\infty}} \leq \alpha_{2}$ and $\left\|r_{a}(t)\right\|_{\mathcal{L}_{\infty}} \leq \alpha_{3}$. Differentiating the equation (39) with respect to time we obtain the following second order differential equation

$$
\begin{aligned}
& \ddot{\tilde{\boldsymbol{u}}}(t)+(\lambda+\sigma) \dot{\tilde{\boldsymbol{u}}}(t)+\left(\lambda \sigma \mathbb{I}_{q}+\gamma \rho(t) B_{m}^{\top} P B_{m} \Lambda\right) \tilde{\boldsymbol{u}}(t)=\gamma \rho(t) B_{m}^{\top} P B_{m} \Lambda K_{1} \boldsymbol{e}^{0}(t) \\
& -\gamma \rho(t) B_{m}^{\top} P A_{m} \boldsymbol{e}(t)-\gamma \dot{\rho}(t) B_{m}^{\top} P \boldsymbol{e}(t)+\lambda \boldsymbol{r}_{a}(t)+\dot{\boldsymbol{r}}_{a}(t)-\lambda \dot{\boldsymbol{f}}(t)-\ddot{\boldsymbol{f}}(t)-\lambda \sigma \boldsymbol{u}^{0}(t)-\sigma \dot{\boldsymbol{u}}^{0}(t) .
\end{aligned}
$$

Since all terms in equation (41) are bounded and continuous functions in time, it can be considered as a second order linear equation with time varying coefficients in $\tilde{\boldsymbol{u}}(t)$. Although it is non-autonomous, it can be still inferred that the adaptation rate $\gamma$ determines the frequency of $\tilde{\boldsymbol{u}}(t)$ and hence the frequency of the control signal $\boldsymbol{u}(t)$, since the ideal control $\boldsymbol{u}^{0}(t)$ is in the low frequency range. Therefore, increasing $\gamma$ increases the oscillations in $\boldsymbol{u}(t)$ as it is the case for the conventional MRAC design. On the other hand $\lambda$ determines the damping ratio. Therefore increasing $\lambda$ suppresses the oscillations in $\tilde{\boldsymbol{u}}(t)$ and hence in the control signal $\boldsymbol{u}(t)$. That is, by selecting a proper value for $\lambda$ the desired performance can be achieved. This is the main difference from the MRAC design, which results when $\lambda=0$.

In the previous subsection we set $\lambda=c_{0} \sqrt{\gamma}$ from the perspective of minimizing the tracking error. The parameter $c_{0}$ and hence $\lambda$ can be selected from the perspective of minimizing $\|\tilde{\boldsymbol{u}}(t)\|_{\mathcal{L}_{\infty}}$. However, the derivation of the bound on $\|\tilde{\boldsymbol{u}}(t)\|_{\mathcal{L}_{\infty}}$ requires the analytic computation of the state transition matrix of non-autonomous system (41), which even if possible, in turn requires the knowledge of entire function $\rho(t)$. This task is not pursuit here. Instead, we minimize the approximate bound on $\|\tilde{\boldsymbol{u}}(t)\|_{\mathcal{L}_{\infty}}$, replacing the function $\rho(t)$ with a positive constant $0<\rho_{0} \leq \alpha_{1}$. This is motivated by the fact that $\rho(t) \leq \alpha_{1}$ for all $t \geq 0$, and we are mainly interested in the asymptotic behavior of $\|\tilde{\boldsymbol{u}}(t)\|_{\mathcal{L}_{\infty}}$ when the parameters $\lambda$ and $\gamma$

are increased. That is, when the influence of the parameters $\lambda$ and $\gamma$ is much greater than the influence of other factors. Taking into account the fact that the initial conditions play a decisive role in the transient behavior of $\tilde{u}(t)$, it is natural to set $\rho_{0}=\rho(0)=\left\|\boldsymbol{x}_{0}\right\|^{2}+\|\boldsymbol{r}(0)\|^{2}+1$. In some applications, when $\boldsymbol{r}(0)=0$ (tracking of a sinusoidal command), it is recommended to use $\|\boldsymbol{r}(t)\|_{\mathcal{L}_{\infty}}^{2}$ in the computation of $\rho_{0}$.

To simplify computations we notice that the matrix $B_{m}^{\top} P B_{m} \Lambda$ is symmetric and positive definite, therefore there exists an orthogonal matrix $T$ such that $D=T B_{m}^{\top} P B_{m} \Lambda T^{\top}$ is diagonal with positive entries 
$d_{i i}, i=1, \ldots, q$. That is, introducing new variables $\boldsymbol{v}^{0}=T \boldsymbol{u}^{0}$ and $\tilde{\boldsymbol{v}}=T \tilde{\boldsymbol{u}}$, we can write the approximate equation in the form

$$
\begin{aligned}
& \ddot{\tilde{\boldsymbol{v}}}(t)+(\lambda+\sigma) \dot{\tilde{\boldsymbol{v}}}(t)+\left(\lambda \sigma \mathbb{I}_{q}+\gamma \rho_{0} D\right) \tilde{\boldsymbol{v}}(t)=\gamma \rho(t) D T^{-\top} K_{1} \boldsymbol{e}^{0}(t)-\gamma \rho(t) T B_{m}^{\top} P A_{m} \boldsymbol{e}(t) \\
& -\gamma \dot{\rho}(t) T B_{m}^{\top} P \boldsymbol{e}(t)+\lambda T \boldsymbol{r}_{a}(t)+\dot{T} \boldsymbol{r}_{a}(t)-\lambda T \dot{\boldsymbol{f}}(t)-T \ddot{\boldsymbol{f}}(t)-\lambda \sigma \boldsymbol{v}^{0}(t)-\sigma \dot{\boldsymbol{v}}^{0}(t) .
\end{aligned}
$$

Therefore, for each component $\tilde{v}_{i}(t), 1=1, \ldots, q$ of the vector $\tilde{\boldsymbol{v}}(t)$ the following norm bound can be written

$$
\begin{aligned}
\left\|\tilde{v}_{i}(t)\right\| & \leq \gamma \alpha_{1} d_{i i}\left\|T^{-\top} K_{1}\right\|_{2}\left\|g_{1 i}(t)\right\|_{\mathcal{L}_{1}}\left\|\boldsymbol{e}^{0}(t)\right\|_{\mathcal{L}_{\infty}}+\gamma \alpha_{1}\left\|T B_{m}^{\top} P A_{m}\right\|_{2}\left\|g_{1 i}(t)\right\|_{\mathcal{L}_{1}}\|\boldsymbol{e}(t)\|_{\mathcal{L}_{\infty}} \\
& +\gamma \alpha_{2}\left\|T B_{m}^{\top} P\right\|_{2}\left\|g_{1 i}(t)\right\|_{\mathcal{L}_{1}}\|\boldsymbol{e}(t)\|_{\mathcal{L}_{\infty}}+\alpha_{3}\|T\|_{2}\left\|g_{2 i}(t)\right\|_{\mathcal{L}_{1}}+\left\|T \boldsymbol{r}_{1}(0)\right\|\left\|g_{1 i}(t)\right\|_{\mathcal{L}_{1}} \\
& +\left\|g_{1 i}(t)\right\|_{\mathcal{L}_{1}}\|\dot{\tilde{\boldsymbol{v}}}(0)\|+\left\|g_{2 i}(t)\right\|_{\mathcal{L}_{1}}\|\tilde{\boldsymbol{v}}(0)\|+\sigma\left\|g_{2 i}(t)\right\|_{\mathcal{L}_{1}}\left\|\boldsymbol{v}^{0}(t)\right\|_{\mathcal{L}_{\infty}}+\left\|g_{3 i}(t)\right\|_{\mathcal{L}_{1}}\|\boldsymbol{f}(t)\|_{\mathcal{L}_{\infty}},
\end{aligned}
$$

where the subscript 2 indicates the induced 2-norm for the corresponding matrices, $g_{1 i}(t), g_{2 i}(t)$ and $g_{3 i}(t)$ are the impulse responses corresponding to the transfer functions $G_{1 i}(s), G_{2 i}(s)$ and $G_{3 i}(s)$, which are defined as

$$
\begin{aligned}
G_{1}(s) & =\frac{1}{s^{2}+2 \zeta_{i} \omega_{i} s+\omega_{i}^{2}} \\
G_{2}(s) & =\frac{s+2 \zeta_{i} \omega_{i}}{s^{2}+2 \zeta_{i} \omega_{i} s+\omega_{i}^{2}} \\
G_{3}(s) & =\frac{s^{2}+2 \zeta_{i} \omega_{i} s}{s^{2}+2 \zeta_{i} \omega_{i} s+\omega_{i}^{2}}
\end{aligned}
$$

for each $i=1, \ldots, q$ with $\omega_{i}=\sqrt{\lambda \sigma+\gamma \rho_{0} d_{i}}$ and $\zeta_{i}=\frac{\lambda+\sigma}{2 \omega_{i}}$. O

Our goal is to find an optimal (or suboptimal) value $\zeta_{0}$ that minimizes the $\mathcal{H}_{\infty}$ norms of these transfer functions independent of the frequency $\omega_{i}$. The rationale behind this is to preserve $\gamma$ in the bounds on the impulse responses in order to be able to cancel $\gamma$ out on the right hand side of (44). This minimization problem for the first two transfer functions is solved in ${ }^{18}$ with the results

$$
\zeta_{0}=0.634,\left\|G_{1 i}(s)\right\|_{\mathcal{H}_{\infty}}=\frac{c_{1}}{\omega_{i}^{2}},\left\|G_{2 i}(s)\right\|_{\mathcal{H}_{\infty}}=\frac{c_{2}}{\omega_{i}},
$$

where $c_{1}=1.0198$ and $c_{2}=1.4162$. On the other hand, the $\mathcal{H}_{\infty}$ norm of $G_{3 i}(s)$ does not depend on $\omega_{i}$, and is given by

$$
\left\|G_{3 i}(s)\right\|_{\mathcal{H}_{\infty}}=\sqrt{1+\frac{2}{4 \zeta_{i}^{2}-1+\sqrt{8 \zeta_{i}^{2}+1}}},
$$

which is a decreasing function in $\zeta_{i}$, and tends to 1 as $\zeta_{i} \rightarrow \infty$. At $\zeta_{i}=\zeta_{0}$ we have $\left\|G_{3 i}(s)\right\|_{\mathcal{H}_{\infty}}=c_{3}=2.514$.

Then taking into account the relationship (see Theorem 4.5 in Ref. ${ }^{23}$ )

$$
\|G(s)\|_{\mathcal{H}_{\infty}} \leq\|g(t)\|_{\mathcal{L}_{1}} \leq 2\|G(s)\|_{\mathcal{H}_{\infty}}
$$

and denoting $d_{0}=\min \left\{d_{11}, \ldots, d_{q q}\right\}>0$, the following bounds readily are obtained

$$
\left\|g_{1 i}(t)\right\|_{\mathcal{L}_{1}} \leq \frac{2 c_{1}}{\lambda \sigma+\gamma \rho_{0} d_{0}},\left\|g_{2 i}(t)\right\|_{\mathcal{L}_{1}} \leq \frac{2 c_{2}}{\sqrt{\lambda \sigma+\gamma \rho_{0} d_{0}}},\left\|g_{3 i}(t)\right\|_{\mathcal{L}_{1}} \leq 2 c_{3}
$$

for the optimal $\zeta_{0}$. We select $\lambda$ to satisfy the equation

$$
\lambda+\sigma=2 \zeta_{0} \sqrt{\lambda \sigma+\gamma \rho_{0} d_{0}},
$$

which can be transformed into the quadratic equation with only root

$$
\lambda=\left(2 \zeta_{0}^{2}-1\right) \sigma+\sqrt{\left(2 \zeta_{0}^{2}-1\right)^{2} \sigma^{2}-\sigma^{2}+4 \zeta_{0}^{2} \gamma \rho_{0} d_{0}},
$$


since $4 \zeta_{0}^{2} \gamma \rho_{0} d_{0}$ is usually much grater than $\sigma^{2}$. It follows from equation (50) that $\lambda$ is asymptotically proportional to $\sqrt{\gamma}$ with the coefficient $c_{0}=2 \zeta_{0} \sqrt{\rho_{0} d_{0}}$ when $\sigma<<\gamma$ (this usually is the case). With this selection of $\lambda$, the bound (44) takes the form

$$
\begin{aligned}
\|\tilde{\boldsymbol{v}}(t)\|_{\mathcal{L}_{\infty}} & \leq \frac{2 \gamma c_{1} \alpha_{1} d^{0}}{\lambda \sigma+\gamma \rho_{0} d_{0}}\left\|T^{-\top}\right\|_{2}\left\|K_{1} \boldsymbol{e}^{0}(t)\right\|_{\mathcal{L}_{\infty}}+\frac{2 \gamma c_{1} \alpha_{1}}{\lambda \sigma+\gamma \rho_{0} d_{0}}\left\|T B_{m}^{\top} P A_{m}\right\|_{2}\|\boldsymbol{e}(t)\|_{\mathcal{L}_{\infty}} \\
& +\frac{2 \gamma c_{1} \alpha_{2}}{\lambda \sigma+\gamma \rho_{0} d_{0}}\left\|T B_{m}^{\top} P\right\|_{2}\|\boldsymbol{e}(t)\|_{\mathcal{L}_{\infty}}+\frac{2 c_{2} \alpha_{3}}{\sqrt{\lambda \sigma+\gamma \rho_{0} d_{0}}}\|T\|_{2}+\frac{2 c_{1}}{\lambda \sigma+\gamma \rho_{0} d_{0}}\left[\left\|T \boldsymbol{r}_{1}(0)\right\|+\|\dot{\tilde{\boldsymbol{v}}}(0)\|\right] \\
& +\frac{2 c_{2}}{\sqrt{\lambda \sigma+\gamma \rho_{0} d_{0}}}\left[\|\tilde{\boldsymbol{v}}(0)\|+\left\|\boldsymbol{v}^{0}(t)\right\|_{\mathcal{L}_{\infty}}\right]+2 c_{3}\|\boldsymbol{f}(t)\|_{\mathcal{L}_{\infty}},
\end{aligned}
$$

where $d^{0}=\max \left\{d_{11}, \ldots, d_{q q}\right\}>0$. Since $\tilde{\boldsymbol{u}}(t)=T^{-1} \tilde{\boldsymbol{v}}(t)$ and $\|\tilde{\boldsymbol{u}}(t)\|_{\mathcal{L}_{\infty}} \leq\|T\|_{2}^{-1}\|\tilde{\boldsymbol{v}}(t)\|_{\mathcal{L}_{\infty}}$, the approximate bound on $\|\boldsymbol{u}(t)\|_{\mathcal{L}_{\infty}}$ takes the form

$$
\begin{aligned}
\|\tilde{\boldsymbol{u}}(t)\|_{\mathcal{L}_{\infty}} & \leq \frac{2 \gamma c_{1} \alpha_{1} d^{0}}{\lambda \sigma+\gamma \rho_{0} d_{0}}\left\|K_{1} \boldsymbol{e}^{0}(t)\right\|_{\mathcal{L}_{\infty}}+\frac{2 \gamma c_{1} \alpha_{1}}{\lambda \sigma+\gamma \rho_{0} d_{0}}\left\|B_{m}^{\top} P A_{m}\right\|_{2}\|\boldsymbol{e}(t)\|_{\mathcal{L}_{\infty}} \\
& +\frac{2 \gamma c_{1} \alpha_{2}}{\lambda \sigma+\gamma \rho_{0} d_{0}}\left\|B_{m}^{\top} P\right\|_{2}\|\boldsymbol{e}(t)\|_{\mathcal{L}_{\infty}}+\frac{2 c_{2} \alpha_{3}}{\lambda \sigma+\sqrt{\gamma \rho_{0} d_{0}}}+\frac{2 c_{1}}{\lambda \sigma+\gamma \rho_{0} d_{0}}\left[\left\|\boldsymbol{r}_{1}(0)\right\|+\|\dot{\tilde{\boldsymbol{u}}}(0)\|++\|\dot{\boldsymbol{f}}(0)\|\right] \\
& +\frac{2 c_{2}}{\sqrt{\lambda \sigma+\gamma \rho_{0} d_{0}}}\left[\|\tilde{\boldsymbol{u}}(0)\|+\|\boldsymbol{f}(0)\|+\left\|\boldsymbol{u}^{0}(t)\right\|_{\mathcal{L}_{\infty}}\right]+2 c_{3}\|\boldsymbol{f}(t)\|_{\mathcal{L}_{\infty}} .
\end{aligned}
$$

We notice that the bound (52) depends on the norm bound of the disturbance term $\boldsymbol{f}(t)$, but not its derivatives.

Taking into account the bounds on $\|\boldsymbol{e}(t)\|_{\mathcal{L}_{\infty}}$ and $\left\|\boldsymbol{e}^{0}(t)\right\|_{\mathcal{L}_{\infty}}$, after some algebra the inequality (52) can be represented in the form

$$
\|\tilde{\boldsymbol{u}}(t)\|_{\mathcal{L}_{\infty}} \leq \beta_{0}+\frac{\beta_{1}}{\sqrt{\gamma}}+\frac{\beta_{2}}{\gamma}
$$

where

$$
\beta_{0}=\frac{2 c_{1} \alpha_{1} d^{0}}{\rho_{0} d_{0}}\left\|K_{1} \boldsymbol{e}^{0}(t)\right\|_{\mathcal{L}_{\infty}}+2 c_{3}\|\boldsymbol{f}(t)\|_{\mathcal{L}_{\infty}}
$$

and depends on the initial parameter estimation error expressed by $\sigma$ and on the external disturbance bound. $\beta_{1}$ and $\beta_{2}$ are readily obtained from (52) and are independent of the design parameters $\lambda$ and $\gamma$. Therefore, last two terms on the right hand side of (53) can be arbitrarily decreased by increasing $\gamma$, when $\lambda$ is selected according to equation (50).

The transient behavior of control signal is given by the following theorem.

Theorem IV.3 Let the system (1) be controlled by the M-MRAC scheme given by (6), (9) and (7), and $\lambda$ satisfies the equation (50). Then for large $\gamma$, the $\mathcal{L}_{\infty}$ norm of the error signals $e(t), e^{0}(t)$ and $\tilde{u}(t)$ can be approximately bounded as follows

$$
\|\tilde{\boldsymbol{u}}(t)\|_{\mathcal{L}_{\infty}} \leq \beta_{0}+o\left(\gamma^{-\frac{1}{2}}\right)
$$

where $\beta_{0}$ is determined by the $\mathcal{L}_{\infty}$ bound of $e^{0}(t)$, given by Theorem IV.2.

Remark IV.1 Although the quantitative analysis of the transient behavior of the adaptive control signal are approximate, the relationship (50), or alternatively

$$
\lambda=2 \zeta_{0} \sqrt{\gamma \rho_{0} d_{0}}
$$

for small $\sigma$, can be used as a rule of thumb when selecting $\lambda$. This selection normally gives a good performance, which can be further improved by searching in the neighborhood of that value. 


\section{Simulation example with $\sigma$-modification}

We demonstrate the performance of M-MRAC on the example from Ref. ${ }^{6}$ with realizations

$$
A=\left[\begin{array}{ll}
0 & 1 \\
2 & 1
\end{array}\right], B=\left[\begin{array}{l}
0 \\
1
\end{array}\right], A_{m}=\left[\begin{array}{cc}
0 & 1 \\
-2 & -3
\end{array}\right], B_{m}=\left[\begin{array}{l}
0 \\
2
\end{array}\right], C=\left[\begin{array}{ll}
2 & 1
\end{array}\right],
$$

which satisfies Assumption II.1 with $\Lambda=0.5, K_{1}=[-4-4]$ and $K_{2}=2$. The external disturbance is a square wave of amplitude 1 and of frequency $0.25 \mathrm{hz}$. In the Lyapunov equation (8) we set $Q=\mathbb{I}_{2}$. The design parameters are chosen as follows $\sigma=0.03, \lambda=\sqrt{2 \gamma\left(x_{0}^{2}+r^{2}+1\right)}$ according to equation (50), where small $\sigma$ is ignored. We run simulations from zero initial conditions for both the system and reference model with a unit step command at time $t=1 \mathrm{sec}$. For $\gamma=1000$, the results are displayed in Figure 1 . The tracking and control signal errors are displayed in Figure 2. It can be seen that good tracking is achieved for both output and control signals, and the later does not exhibit any high frequency oscillations, which is unavoidable for the conventional MRAC design. This can be seen from Figure 3
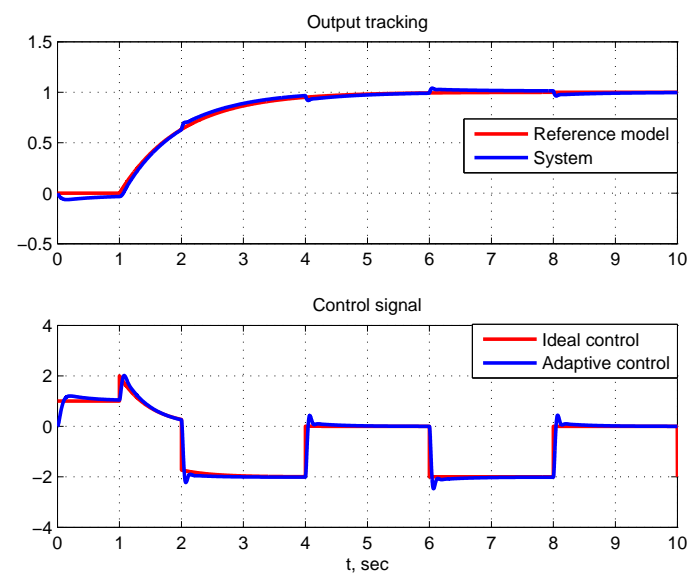

Figure 1. M-MRAC performance with $\sigma$-modification for $\gamma=1000$.
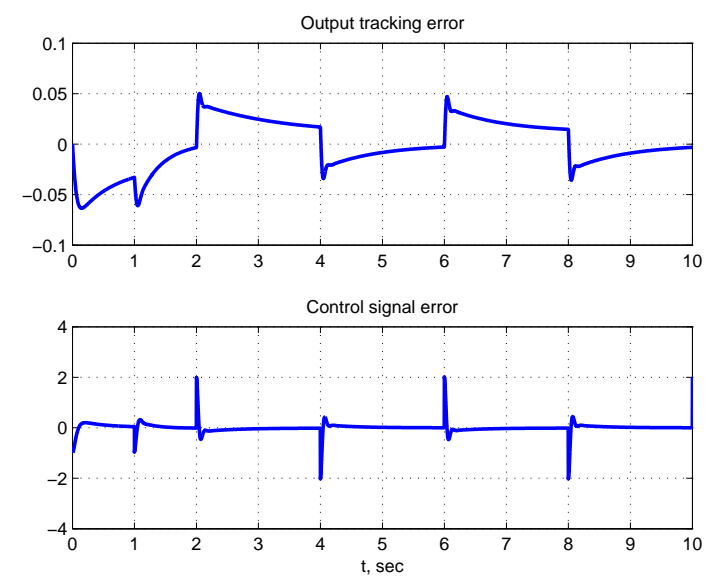

Figure 2. M-MRAC error signals with $\sigma$-modification for $\gamma=1000$.

When we increase the adaptation rate to $\gamma=10000$, the tracking error magnitude decreases as predicted. The control error bound is mainly determined by the parameter initialization error and does not noticeably change. This can be observed from Figure 4.

To test the algorithm for the differentiable disturbance signals, we replace the square wave with a sinusoid of the same magnitude and frequency. This further decreases the tracking error bound as predicted by 

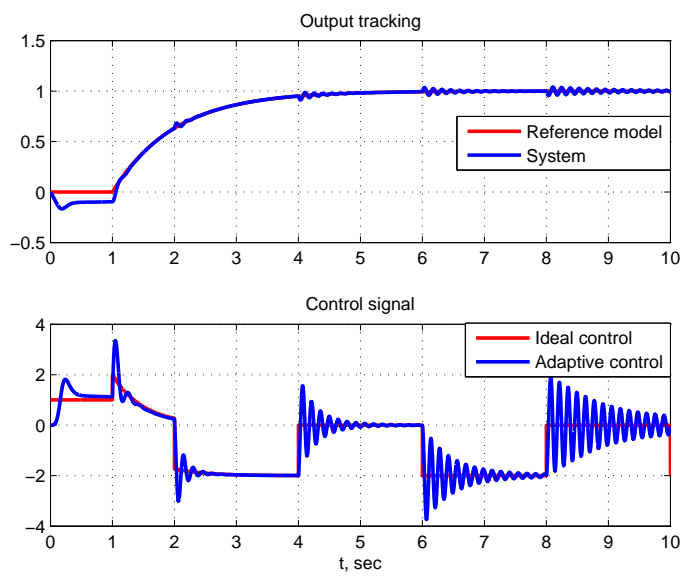

Figure 3. MRAC performance with $\sigma$-modification for $\gamma=1000$.
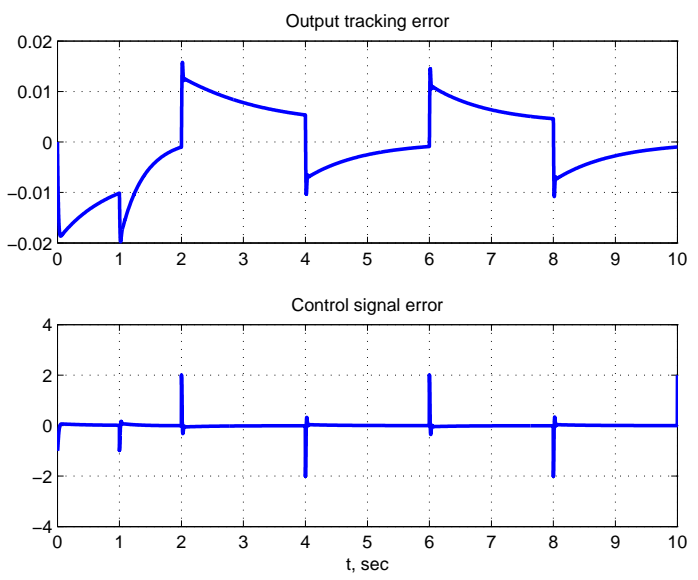

Figure 4. M-MRAC error signals with $\sigma$-modification for $\gamma=10000$.

Theorem IV.2 and as seen in Figure 5. It can be also observed that the control error is substantially smaller, since the parameter estimates are not being reset contrary to the case of a square wave.

\section{Performance analysis of M-MRAC with $e$-modification}

In this section, we set

$$
\begin{aligned}
\Psi_{1}\left(\boldsymbol{x}, \boldsymbol{e}, \hat{K}_{1}\right) & =-\|\boldsymbol{e}\| \hat{K}_{1} \\
\Psi_{2}\left(\boldsymbol{r}, \boldsymbol{e}, \hat{K}_{2}\right) & =-\|\boldsymbol{e}\| \hat{K}_{2} \\
\Psi_{3}(\boldsymbol{e}, \hat{\boldsymbol{f}}) & =-\|\boldsymbol{e}\| \hat{\boldsymbol{f}}
\end{aligned}
$$

and provide asymptotic and transient analysis of the M-MRAC architecture.

\section{A. Stability}

The stability of system (1) controlled by the M-MRAC scheme given by (6), (9) and (7) with (57) is proved similar to the previous section using the candidate Lyapunov function $V(t)$ (18). In this case, its derivative 

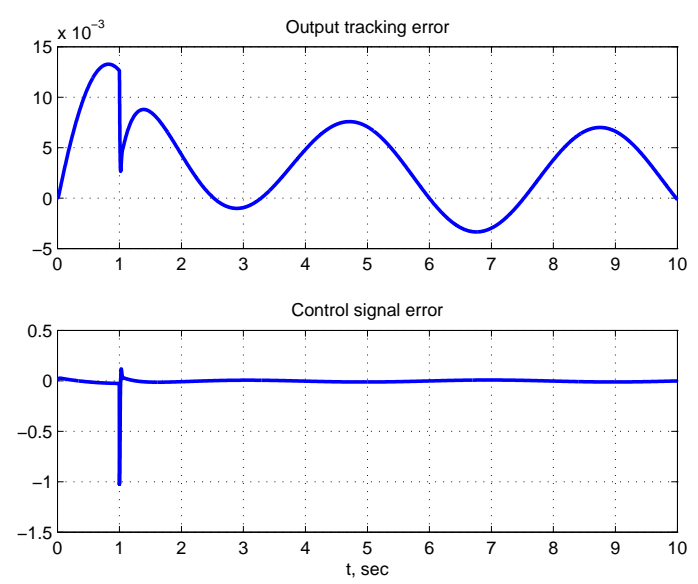

Figure 5. M-MRAC error signals with $\sigma$-modification for the sinusoidal disturbance $(\gamma=10000)$.

computed along the trajectories of the systems (10) and (7) with (57) takes the form

$$
\begin{aligned}
\dot{V}(t) & =-e^{\top}(t) Q \boldsymbol{e}(t)-2 \lambda e^{\top}(t) P \boldsymbol{e}(t)+2 e^{\top}(t) P B_{m} \Lambda(\overline{\boldsymbol{f}}-\boldsymbol{f}(t)) \\
& -2 \frac{\|\boldsymbol{e}(t)\|}{\gamma} \operatorname{tr}\left(\tilde{K}_{1}^{\top}(t) \hat{K}_{1}(t) \Lambda+\tilde{K}_{2}^{\top}(t) \hat{K}_{2}(t) \Lambda+\tilde{\boldsymbol{f}}^{\top}(t) \hat{\boldsymbol{f}}(t) \Lambda\right),
\end{aligned}
$$

which upon completion of the squares satisfies the inequality

$$
\dot{V}(t) \leq\|\boldsymbol{e}(t)\|\left(-a_{1}\|e(t)\|+c\right)-\frac{\sigma}{\gamma} \lambda_{\min }(\Lambda)\|\boldsymbol{e}(t)\|\left(\left\|\tilde{K}_{1}(t)\right\|_{F}^{2}+\left\|\tilde{K}_{2}(t)\right\|_{F}^{2}+\|\tilde{\boldsymbol{f}}(t)\|^{2}\right),
$$

where the same notations are used as in the previous case. Again, choosing $Q$ such that $\lambda_{\min }(Q)-1 \geq 0$, we conclude that $\dot{V}(t)$ is negative semi-definite outside the compact set

$$
\Omega_{2}=\left\{\left(\boldsymbol{e}, \tilde{K}_{1}, \tilde{K}_{2}, \tilde{\boldsymbol{f}}\right): a_{1}\|\boldsymbol{e}\| \leq c\right\} .
$$

which implies that all closed-loop signals are uniformly ultimately bounded as in the previous case.

\section{B. Transient behavior of the tracking error}

As in the previous case, the inequality (22) holds. It follows that the trajectories stay inside the Lyapunov level set

$$
L_{2}=\left\{\left(\boldsymbol{e}, \tilde{K}_{1}, \tilde{K}_{2}, \tilde{\boldsymbol{f}}\right): V\left(\boldsymbol{e}, \tilde{K}_{1}, \tilde{K}_{2}, \tilde{\boldsymbol{d}}\right)=\lambda_{\max }(P) \frac{c^{2}}{a_{1}^{2}}+\frac{1}{\gamma} k_{2}^{*}\right\}
$$

where $k_{2}^{*}$ is defined in (22). Therefore, the following conservative bound can be derived

$$
\|\boldsymbol{e}(t)\|_{\mathcal{L}_{\infty}} \leq \sqrt{\frac{\lambda_{\max }(P)}{\lambda_{\min }(P)} \frac{c^{2}}{a_{1}^{2}}+\frac{1}{\gamma \lambda_{\min }(P)} k_{2}^{*}} .
$$

It follows from the inequality $(71)$ that $\|\boldsymbol{e}(t)\|_{\mathcal{L}_{\infty}}$ can be decreased as desired by increasing $\gamma$. On the other hand the actual tracking error satisfies the norm bound

$$
\left\|\boldsymbol{e}^{0}(t)\right\|_{\mathcal{L}_{\infty}} \leq\left(1+k_{m} \lambda\right) \sqrt{\frac{\lambda_{\max }(P)}{\lambda_{\min }(P)} \frac{c^{2}}{a_{1}^{2}}+\frac{1}{\gamma \lambda_{\min }(P)} k_{2}^{*}},
$$

which results in the limiting equality

$$
\lim _{\gamma \rightarrow \infty}\left\|\boldsymbol{e}^{0}(t)\right\|_{\mathcal{L}_{\infty}} \leq k_{m} \sqrt{\frac{c_{0}}{\lambda_{\min }(P)} k_{2}^{*}},
$$


when we set $\lambda=c_{0} \sqrt{\gamma}$. Clearly, this asymptotic bound can be further decrease by increasing $\lambda_{\min }(P)$. We notice that the tracking error bound in the case of $e$-modification is comparable with the similar bound in $\sigma$ - modification case when the disturbance term has a bounded derivative.

\section{Transient behavior of the control signal}

To reflect the changes in the control signal transient behavior we notice that the term $\sigma \boldsymbol{u}(t)$ in the equation (39) is replaced with $\|\boldsymbol{e}(t)\| \boldsymbol{u}(t)$, which gives rise additional terms in the $\tilde{\boldsymbol{u}}(t)$ dynamics, which in this case can be written as

$$
\begin{aligned}
& \ddot{\tilde{\boldsymbol{u}}}(t)+[\lambda+\|\boldsymbol{e}(t)\|] \dot{\tilde{\boldsymbol{u}}}(t)+\left[\gamma \rho(t) B_{m}^{\top} P B_{m} \Lambda-\lambda\|\boldsymbol{e}(t)\| \mathbb{I}_{q}+\frac{1}{\|\boldsymbol{e}(t)\|} \boldsymbol{e}^{\top}(t) A_{m} \boldsymbol{e}(t) \mathbb{I}_{q}-\frac{\lambda}{\|\boldsymbol{e}(t)\|} \boldsymbol{e}^{\top}(t) \boldsymbol{e}(t) \mathbb{I}_{q}\right. \\
& \left.+\frac{1}{\|\boldsymbol{e}(t)\|} \boldsymbol{e}^{\top}(t) B_{m} \Lambda K_{1} \boldsymbol{e}^{0}(t) \mathbb{I}_{q}+\frac{1}{\|\boldsymbol{e}(t)\|} \boldsymbol{u}^{0}(t) \boldsymbol{e}^{\top}(t) B_{m} \Lambda\right] \tilde{\boldsymbol{u}}(t)+\frac{1}{\|\boldsymbol{e}(t)\|} \tilde{\boldsymbol{u}}(t) \boldsymbol{e}^{\top}(t) B_{m} \Lambda \tilde{\boldsymbol{u}}(t) \\
& =\gamma \rho(t) B_{m}^{\top} P B_{m} \Lambda K_{1} \boldsymbol{e}^{0}(t)-\gamma \rho(t) B_{m}^{\top} P A_{m} \boldsymbol{e}(t)-\gamma \dot{\rho}(t) B_{m}^{\top} P \boldsymbol{e}(t)+\lambda \boldsymbol{r}_{a}(t)+\dot{\boldsymbol{r}}_{a}(t)-\lambda \dot{\boldsymbol{f}}(t)-\ddot{\boldsymbol{f}}(t) \\
& -\|\boldsymbol{e}(t)\|\left[\lambda \boldsymbol{u}^{0}(t)+\dot{\boldsymbol{u}}^{0}(t)\right]-\frac{1}{\|\boldsymbol{e}(t)\|}\left[\boldsymbol{e}^{\top}(t) A_{m} \boldsymbol{e}(t)-\lambda \boldsymbol{e}^{\top}(t) \boldsymbol{e}(t)+\boldsymbol{e}^{\top}(t) B_{m} \Lambda K_{1} \boldsymbol{e}^{0}(t)\right] \boldsymbol{u}^{0}(t) .
\end{aligned}
$$

The analysis of equation (63) is more complicated that that of the equation (41), since it is nonlinear in $\tilde{\boldsymbol{u}}(t)$. When the nonlinear term $\frac{1}{\|\boldsymbol{e}(t)\|} \tilde{\boldsymbol{u}}(t) \boldsymbol{e}^{\top}(t) B_{m} \Lambda \tilde{\boldsymbol{u}}(t)$ can be neglected, the damping of the signal $\tilde{\boldsymbol{u}}(t)$ for large $\gamma$ is determined by the parameter $\lambda$, since $\boldsymbol{e}(t)$ is of the order of $\gamma^{-1 / 2}$. On the other hand, for $\lambda=c_{0} \sqrt{\gamma}$, the frequency of $\tilde{\boldsymbol{u}}(t)$ is determined by the dominant term $\gamma \rho(t) B_{m}^{\top} P B_{m} \Lambda$, since the remaining terms are either in the order of $\gamma^{-1 / 2}$ or $\gamma^{0}$. Hence the approximate equation (42) in this case can be written in the form

$$
\begin{aligned}
& \ddot{\tilde{\boldsymbol{v}}}(t)+\lambda \dot{\tilde{\boldsymbol{v}}}(t)+\gamma \rho_{0} D \tilde{\boldsymbol{v}}(t)=\gamma \rho(t) D T^{-\top} K_{1} \boldsymbol{e}^{0}(t)-\gamma \rho(t) T B_{m}^{\top} P A_{m} \boldsymbol{e}(t) \\
& -\gamma \dot{\rho}(t) T B_{m}^{\top} P \boldsymbol{e}(t)+\lambda T \boldsymbol{r}_{a}(t)+\dot{T} \boldsymbol{r}_{a}(t)-\lambda T \boldsymbol{f}(t)-T \ddot{\boldsymbol{f}}(t)-\|\boldsymbol{e}(t)\|\left[\lambda \boldsymbol{u}^{0}(t)+\dot{\boldsymbol{u}}^{0}(t)\right] \\
& -\frac{1}{\|\boldsymbol{e}(t)\|}\left[\boldsymbol{e}^{\top}(t) A_{m} \boldsymbol{e}(t)-\lambda \boldsymbol{e}^{\top}(t) \boldsymbol{e}(t)+\boldsymbol{e}^{\top}(t) B_{m} \Lambda K_{1} \boldsymbol{e}^{0}(t)\right] \boldsymbol{u}^{0}(t),
\end{aligned}
$$

and the approximate bound takes the form

$$
\begin{aligned}
\|\tilde{\boldsymbol{u}}(t)\|_{\mathcal{L}_{\infty}} & \leq \frac{2 \gamma c_{1} \alpha_{1} d^{0}}{\gamma \rho_{0} d_{0}}\left\|K_{1} \boldsymbol{e}^{0}(t)\right\|_{\mathcal{L}_{\infty}}+\frac{2 \gamma c_{1} \alpha_{1}}{\gamma \rho_{0} d_{0}}\left\|B_{m}^{\top} P A_{m}\right\|_{2}\|\boldsymbol{e}(t)\|_{\mathcal{L}_{\infty}}+\frac{2 \gamma c_{1} \alpha_{2}}{\gamma \rho_{0} d_{0}}\left\|B_{m}^{\top} P\right\|_{2}\|\boldsymbol{e}(t)\|_{\mathcal{L}_{\infty}} \\
& +\frac{2 c_{2} \alpha_{3}}{\sqrt{\gamma \rho_{0} d_{0}}}+\frac{2 c_{1}}{\gamma \rho_{0} d_{0}}\left[\left\|\boldsymbol{r}_{a}(0)\right\|+\|\dot{\tilde{\boldsymbol{u}}}(0)\|+\|\dot{\boldsymbol{f}}(0)\|\right]+\frac{2 c_{2}}{\sqrt{\gamma \rho_{0} d_{0}}}[\|\tilde{\boldsymbol{u}}(0)\|+\|\boldsymbol{f}(0)\|]+2 c_{3}\|\boldsymbol{f}(t)\|_{\mathcal{L}_{\infty}} \\
& +\frac{2 c_{2}}{\sqrt{\gamma \rho_{0} d_{0}}}\|\boldsymbol{e}(t)\|_{\mathcal{L}_{\infty}}\left\|\boldsymbol{u}^{0}(t)\right\|_{\mathcal{L}_{\infty}}+\frac{2 c_{1}}{\gamma \rho_{0} d_{0}}\left\|A_{m} \boldsymbol{e}(t)-\lambda \boldsymbol{e}(t)+B_{m} \Lambda K_{1} \boldsymbol{e}^{0}(t)\right\|_{\mathcal{L}_{\infty}}\left\|\boldsymbol{u}^{0}(t)\right\|_{\mathcal{L}_{\infty}}, \quad(65)
\end{aligned}
$$

where now we set $\lambda=2 \zeta_{0} \sqrt{\gamma \rho_{0} d_{0}}$. It is easy to see, that the signal $\|\tilde{\boldsymbol{u}}(t)\|_{\mathcal{L}_{\infty}}$ again satisfies the approximate bound (53).

\section{Simulation example with $e$-modification}

Now we consider the simulation example of the previous section with the adaptive laws given by (7) and (57). For $\gamma=1000$ the performance of M-MRAC architecture is displayed in Figure 6, which is similar to the performance with $\sigma$-modification. Generated error signals are also similar to those in the previous case, as can be seen from Figure (7).

Increasing the adaptation rate to $\gamma=10000$ and introducing sinusoidal disturbance have effects similar to the those in previous case, as can be observed from Figures 8 and 9. 

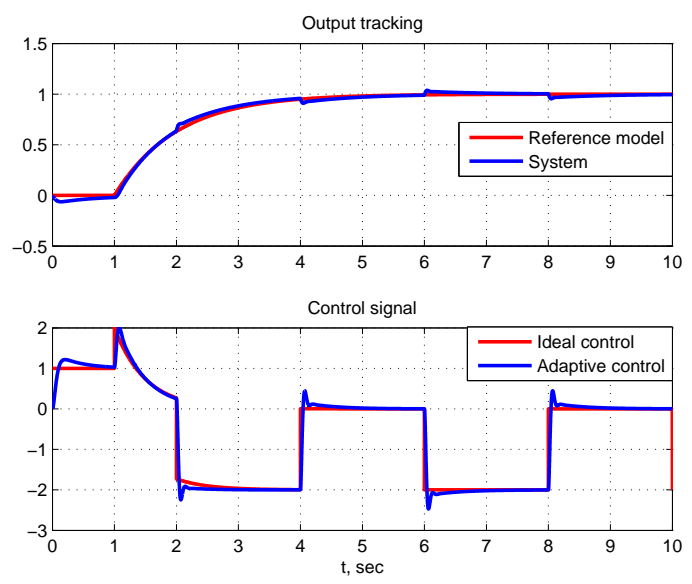

Figure 6. M-MRAC performance with $e$-modification for $\gamma=1000$.
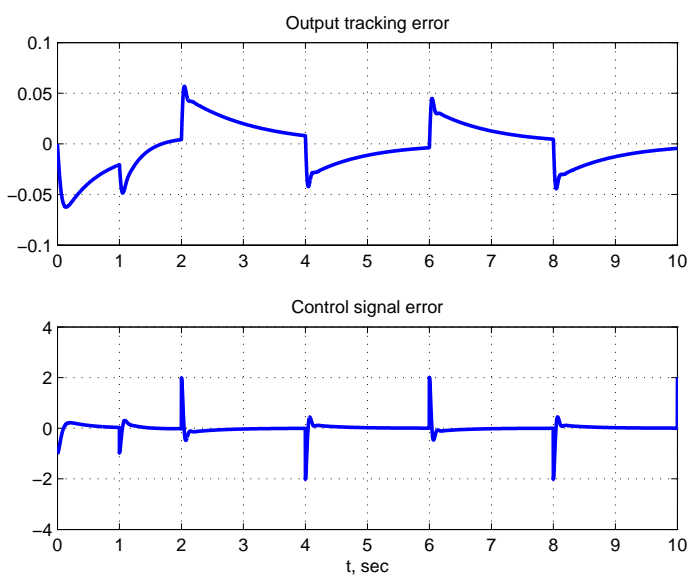

Figure 7. M-MRAC error signals with $e$-modification for $\gamma=1000$.
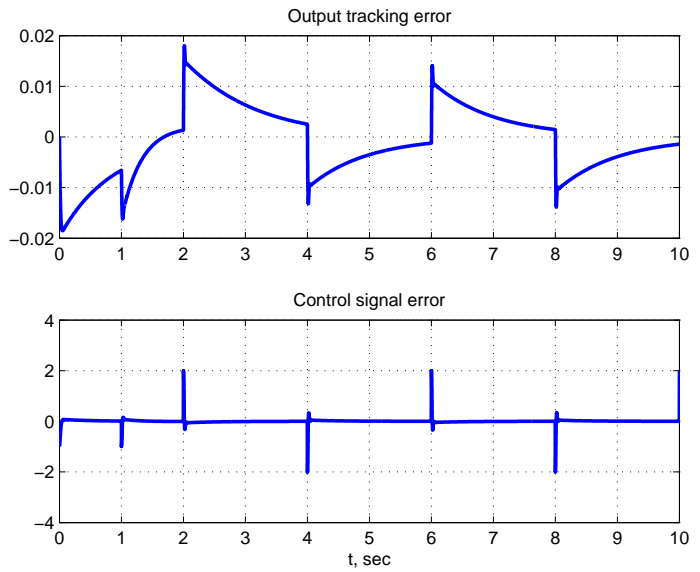

Figure 8. M-MRAC error signals with $e$-modification for $\gamma=10000$. 

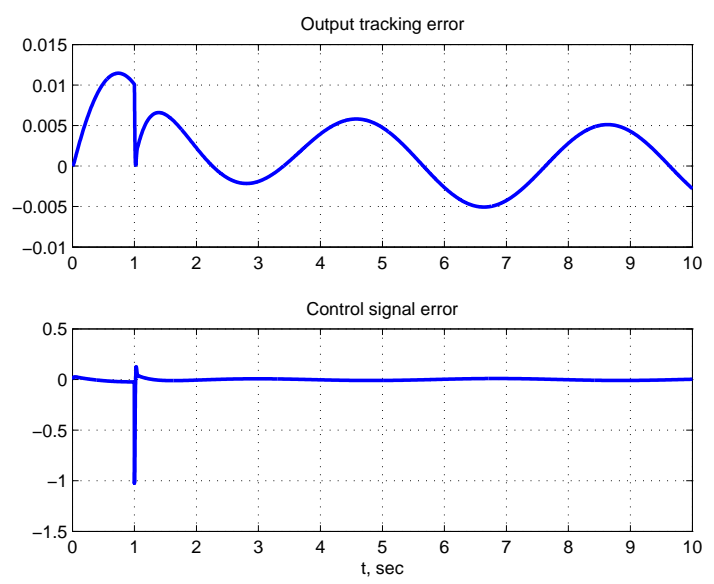

Figure 9. M-MRAC error signals with $e$-modification for the sinusoidal disturbance $(\gamma=10000)$.

\section{Performance analysis of M-MRAC with projection operator}

When the projection operator is used for the robustness of the adaptive laws, the functions $\Psi_{i}(\cdot)$ have the form $\Psi_{i}(\cdot)=H_{i}(\cdot) s_{i}(\cdot)$ for each $i=1,2,3$, where the functions $H_{i}(\cdot)$ are defined as

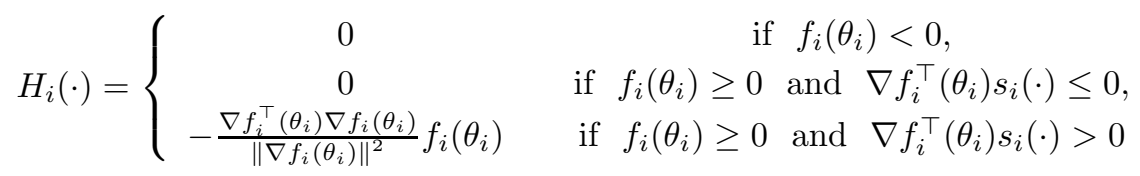

with the notation $\nabla f\left(\theta_{i}\right)=\frac{\partial f\left(\theta_{i}\right)}{\partial \theta_{i}}\left(\theta_{1}=\hat{K}_{1}, \theta_{2}=\hat{K}_{2}, \theta_{3}=\hat{f}\right)$, the smooth convex functions $f_{i}\left(\theta_{i}\right)$ are defined as

$$
f_{i}\left(\theta_{i}\right)=\frac{\operatorname{tr}\left(\theta_{i}^{\top} \theta_{i}\right)-\theta_{i}^{* 2}}{\epsilon_{\theta_{i}} \theta_{i}^{* 2}}
$$

with $\theta_{i}^{*}$ denoting the norm bound imposed on the parameter matrix $\theta_{i}$ and $\epsilon_{\theta_{i}}$ denoting the convergence tolerance, and the functions $g_{i}(\cdot)$ have the form

$$
\begin{aligned}
s_{1}(\cdot) & =-\gamma B_{m}^{\top} \text { Pex } \boldsymbol{x}^{\top} \\
s_{2}(\cdot) & =-\gamma B_{m}^{\top} \text { Per } \\
s_{3}(\cdot) & =-\gamma B_{m}^{\top} P \boldsymbol{e} .
\end{aligned}
$$

\section{A. Stability}

The derivative of the candidate Lyapunov function $V(t)(18)$ in this case satisfies the inequality

$$
\dot{V}(t) \leq-e^{\top}(t) Q \boldsymbol{e}(t)-2 \lambda e^{\top}(t) P \boldsymbol{e}(t)+2 e^{\top}(t) P B_{m} \Lambda(\overline{\boldsymbol{f}}-\boldsymbol{f}(t)),
$$

which is based on the properties of the projection operator. ${ }^{15}$ It can be upper bounded as

$$
\dot{V}(t) \leq\|\boldsymbol{e}(t)\|\left[-a_{2}\|\boldsymbol{e}(t)\|+2 f_{*}\right],
$$

where the same notations are used as in the previous cases. It follows that $\dot{V}(t)$ is negative semi-definite outside the compact set

$$
\Omega_{3}=\left\{\left(\boldsymbol{e}, \tilde{K}_{1}, \tilde{K}_{2}, \tilde{\boldsymbol{f}}\right): a_{2}\|\boldsymbol{e}\| \leq 2 f_{*}\right\} .
$$

which implies that all closed-loop signals are uniformly ultimately bounded as in the previous cases. 


\section{B. Transient behavior of the tracking error}

As in the previous case, the inequality (22) holds. Therefore, it follows that the trajectories stay inside the Lyapunov level set

$$
L_{3}=\left\{\left(\boldsymbol{e}, \tilde{K}_{1}, \tilde{K}_{2}, \tilde{\boldsymbol{f}}\right): V\left(\boldsymbol{e}, \tilde{K}_{1}, \tilde{K}_{2}, \tilde{\boldsymbol{d}}\right)=\lambda_{\max }(P) \frac{4 f_{*}^{2}}{a_{2}^{2}}+\frac{1}{\gamma} k_{2}^{*}\right\},
$$

where $k_{2}^{*}$ is defined in (22). Hence, the following conservative bound can be derived

$$
\|\boldsymbol{e}(t)\|_{\mathcal{L}_{\infty}} \leq \sqrt{\frac{\lambda_{\max }(P)}{\lambda_{\min }(P)} \frac{4 f_{*}^{2}}{a_{2}^{2}}+\frac{1}{\gamma \lambda_{\min }(P)} k_{2}^{*}} .
$$

It follows from the inequality (71) that $\|\boldsymbol{e}(t)\|_{\mathcal{L}_{\infty}}$ can be decreased as desired by increasing $\gamma$. On the other hand the actual tracking error satisfies the norm bound

$$
\left\|e^{0}(t)\right\|_{\mathcal{L}_{\infty}} \leq\left(1+k_{m} \lambda\right) \sqrt{\frac{\lambda_{\max }(P)}{\lambda_{\min }(P)} \frac{4 f_{*}^{2}}{a_{2}^{2}}+\frac{1}{\gamma \lambda_{\min }(P)} k_{2}^{*}},
$$

which results in the limiting equality

$$
\lim _{\gamma \rightarrow \infty}\left\|\boldsymbol{e}^{0}(t)\right\|_{\mathcal{L}_{\infty}} \leq k_{m} \sqrt{\frac{c_{0}}{\lambda_{\min }(P)} k_{2}^{*}},
$$

when we set $\lambda=c_{0} \sqrt{\gamma}$. Clearly, this asymptotic bound can be further decrease by increasing $\lambda_{\min }(P)$. We notice that the tracking error bound in this case is comparable with the bound in the case of $e$-modification.

\section{Transient behavior of the control signal}

We notice that

$$
\Psi_{1}\left(\boldsymbol{x}(t), \boldsymbol{e}(t), \hat{K}_{1}(t)\right) \boldsymbol{x}(t)+\Psi_{2}\left(\boldsymbol{r}(t), \boldsymbol{e}(t), \hat{K}_{2}(t)\right) \boldsymbol{r}(t)+\Psi_{3}(\boldsymbol{e}(t), \hat{\boldsymbol{f}}(t))=-\gamma H(t) B_{m}^{\top} P \boldsymbol{e}(t),
$$

where

$$
H(t)=H_{1}\left(\hat{K}_{1}(t)\right) \boldsymbol{x}^{\top}(t) \boldsymbol{x}(t)+H_{2}\left(\hat{K}_{2}(t)\right) \boldsymbol{r}^{\top}(t) \boldsymbol{r}(t)+H_{3}(\hat{\boldsymbol{f}}(t)) .
$$

It is easy to see that the matrix $H(t) \in \mathbb{R}^{q \times q}$ is positive semi-definite for each $t>0$. Moreover, it has a bounded derivative, since the projection operator is smooth and keeps the parameter estimates bounded. ${ }^{15}$ Therefore, the equation (39) in this case takes the form

$$
\dot{\tilde{\boldsymbol{u}}}(t)=-\gamma \Theta(t) B_{m}^{\top} P \boldsymbol{e}(t)+\boldsymbol{r}_{a}(t)-\dot{\boldsymbol{f}}(t, \boldsymbol{x}),
$$

where the matrix $\Theta(t)=\rho(t) \mathbb{I}_{q \times q}+H(t)$ is bounded and has a bounded time derivative. $\tilde{\boldsymbol{u}}(t)$ dynamics take the form

$$
\begin{aligned}
& \ddot{\tilde{\boldsymbol{u}}}(t)+\lambda \dot{\tilde{\boldsymbol{u}}}(t)+\gamma \Theta(t) B_{m}^{\top} P B_{m} \Lambda \tilde{\boldsymbol{u}}(t)=\gamma \Theta(t) B_{m}^{\top} P B_{m} \Lambda K_{1} \boldsymbol{e}^{0}(t)-\gamma \Theta(t) B_{m}^{\top} P A_{m} \boldsymbol{e}(t) \\
& -\gamma \dot{\Theta}(t) B_{m}^{\top} P \boldsymbol{e}(t)+\lambda \boldsymbol{r}_{a}(t)+\dot{\boldsymbol{r}}_{a}(t)-\lambda \dot{\boldsymbol{f}}(t)-\ddot{\boldsymbol{f}}(t) .
\end{aligned}
$$

It is easy to see that the damping of the signal $\tilde{\boldsymbol{u}}(t)$ is determined by the parameter $\lambda$, and the frequency of it is determined by $\gamma \Theta(t) B_{m}^{\top} P B_{m} \Lambda$. Since the initial parameter estimates are chosen inside the compact set defined by the projection operator, $H(0)=0$. Therefore, the approximate equation (42) in this case can be written in the form

$$
\begin{aligned}
& \ddot{\tilde{\boldsymbol{v}}}(t)+\lambda \dot{\tilde{\boldsymbol{v}}}(t)+\gamma \rho_{0} D \tilde{\boldsymbol{v}}(t)=\gamma \Theta(t) D T^{-\top} K_{1} \boldsymbol{e}^{0}(t)-\gamma \Theta(t) T B_{m}^{\top} P A_{m} \boldsymbol{e}(t) \\
& -\gamma \dot{\Theta}(t) T B_{m}^{\top} P \boldsymbol{e}(t)+\lambda T \boldsymbol{r}_{a}(t)+\dot{T} \boldsymbol{r}_{a}(t)-\lambda T \dot{\boldsymbol{f}}(t)-T \ddot{\boldsymbol{f}}(t),
\end{aligned}
$$

The approximate bound takes the form

$$
\begin{aligned}
\|\tilde{\boldsymbol{u}}(t)\|_{\mathcal{L}_{\infty}} & \leq \frac{2 \gamma c_{1} \delta_{1} d^{0}}{\gamma \rho_{0} d_{0}}\left\|K_{1} \boldsymbol{e}^{0}(t)\right\|_{\mathcal{L}_{\infty}}+\frac{2 \gamma c_{1} \delta_{1}}{\gamma \rho_{0} d_{0}}\left\|B_{m}^{\top} P A_{m}\right\|_{2}\|\boldsymbol{e}(t)\|_{\mathcal{L}_{\infty}}+\frac{2 \gamma c_{1} \delta_{2}}{\gamma \rho_{0} d_{0}}\left\|B_{m}^{\top} P\right\|_{2}\|\boldsymbol{e}(t)\|_{\mathcal{L}_{\infty}} \\
& +\frac{2 c_{2} \alpha_{3}}{\sqrt{\gamma \rho_{0} d_{0}}}+\frac{2 c_{1}}{\gamma \rho_{0} d_{0}}\left[\left\|\boldsymbol{r}_{a}(0)\right\|+\|\dot{\tilde{\boldsymbol{u}}}(0)\|+\|\dot{\boldsymbol{f}}(0)\|\right]+\frac{2 c_{2}}{\sqrt{\gamma \rho_{0} d_{0}}}[\|\tilde{\boldsymbol{u}}(0)\|+\|\boldsymbol{f}(0)\|]+2 c_{3}\|\boldsymbol{f}(t)\|_{\mathcal{L}_{\infty}},
\end{aligned}
$$

where $\delta_{1}>0$ and $\delta_{2}>0$ are the norm bounds on the matrix $\Theta(t)$ and its derivative respectively, and $\lambda=2 \zeta_{0} \sqrt{\gamma \rho_{0} d_{0}}$. Therefore, the control signal error $\|\tilde{\boldsymbol{u}}(t)\|_{\mathcal{L}_{\infty}}$ satisfies the approximate bound (53). 


\section{Simulation example with the projection operator}

We apply M-MRAC algorithm with the projection based adaptive laws to the same system considered in the previous sections. For $\gamma=1000$ the performance of M-MRAC architecture is displayed in Figure 10, and the corresponding error signals are presented in Figure 11. A similar performance can be observed in this case as well.
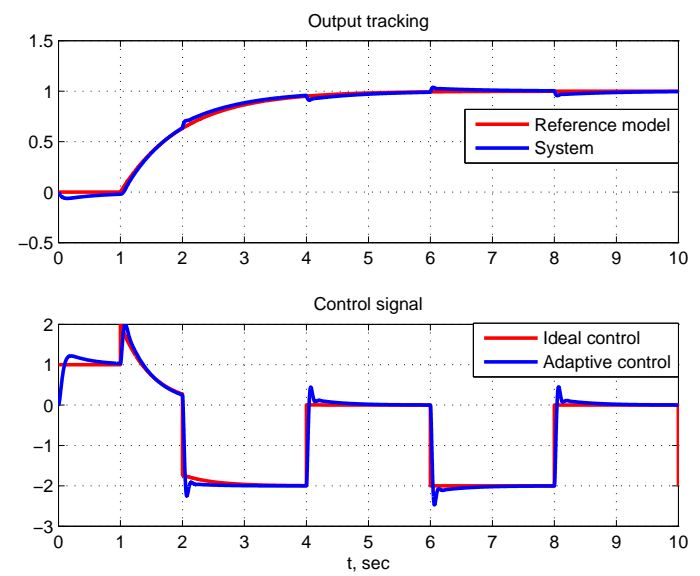

Figure 10. M-MRAC performance with projection operator for $\gamma=1000$.
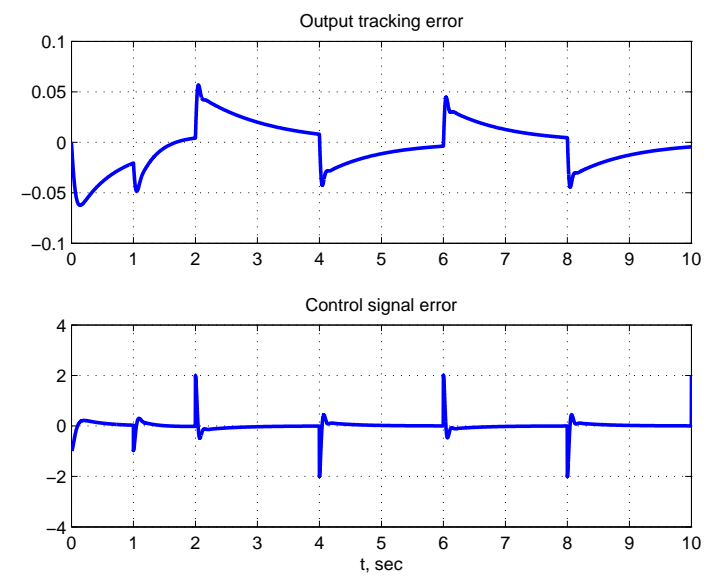

Figure 11. M-MRAC error signals with projection operator for $\gamma=1000$.

Again, increasing the adaptation rate improves the performance (see Figure 12), and the smooth disturbance produces smaller error signals as in previous cases (see Figure 13).

\section{Avoiding bursting}

It is well known that decreasing $\sigma$ in the MRAC framework with $\sigma$-modification results in the bursting of the tracking error, when the input signal is not sufficiently rich. ${ }^{6}$ In particular, this happens in regulation problems, when small $\sigma$ is selected in order to obtain a small residual tracking error. Here, we show that the M-MRAC architecture unlike the conventional MRAC design can avoid this unwanted phenomena by the proper selection of the design parameters.

To see this we set $\boldsymbol{r}(t)=0$ and $\boldsymbol{f}(t)=0$. Then the closed-loop system for the MRAC design takes the 

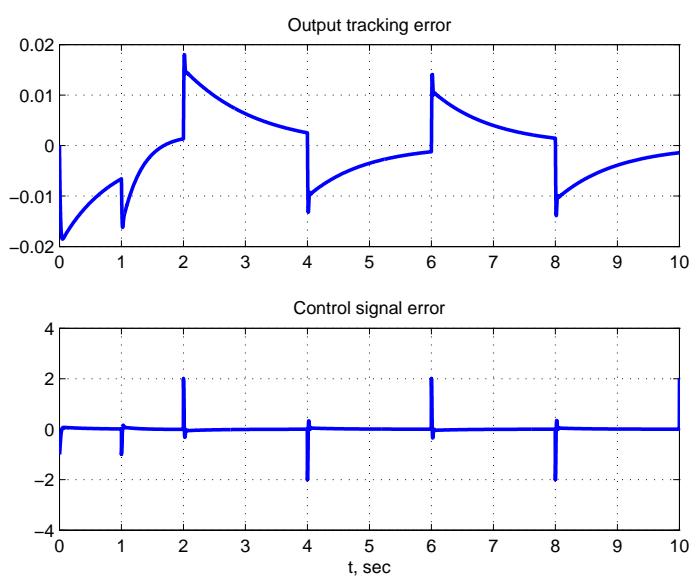

Figure 12. M-MRAC error signals with projection operator for $\gamma=10000$.
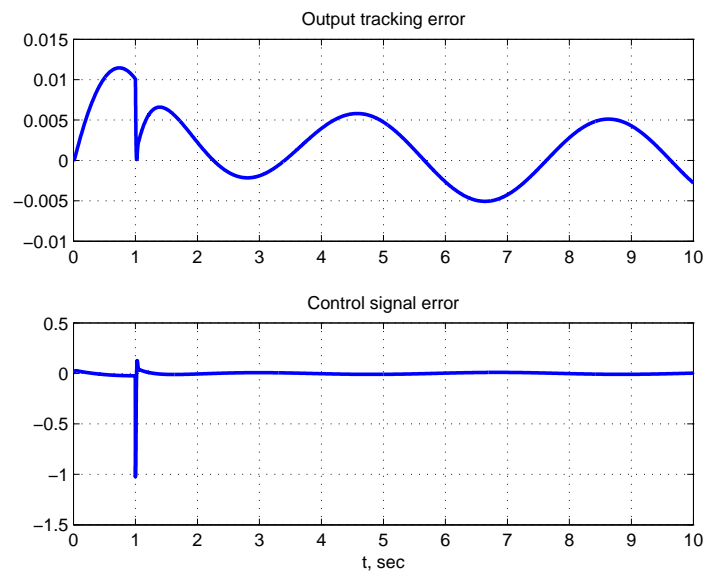

Figure 13. M-MRAC error signals with projection operator for the sinusoidal disturbance $(\gamma=10000)$.

form

$$
\begin{aligned}
\dot{\boldsymbol{x}}^{0}(t) & =A_{m} x^{0}(t) \\
\dot{\boldsymbol{x}}(t) & =A \boldsymbol{x}(t)+B\left[\hat{K}_{1}(t) \boldsymbol{x}(t)+\hat{\boldsymbol{f}}(t)\right] \\
\dot{\hat{K}}_{1}(t) & =-\gamma B_{m}^{\top} P\left[\boldsymbol{x}(t)-\boldsymbol{x}^{0}(t)\right] \boldsymbol{x}^{\top}(t)-\sigma \hat{K}_{1}(t) \\
\dot{\hat{K}}_{2}(t) & =-\sigma \hat{K}_{2}(t) \\
\dot{\hat{\boldsymbol{f}}}(t) & =-\gamma B_{m}^{\top} P\left[\boldsymbol{x}(t)-\boldsymbol{x}^{0}(t)\right]-\sigma \hat{\boldsymbol{f}}(t) .
\end{aligned}
$$

This system obviously has an equilibrium at $\boldsymbol{x}^{0}(t)=0, \boldsymbol{x}=0, \hat{K}_{1}=0, \hat{K}_{2}=0 \hat{\boldsymbol{f}}=0$, the stability of which we are interested in. Linearizing the system (78) around the this equilibrium results in

$$
\left[\begin{array}{c}
\dot{\Delta \boldsymbol{x}^{0}(t)} \\
\dot{\Delta} \boldsymbol{x}(t) \\
\dot{\Delta} \hat{K}_{1}(t) \\
\Delta \hat{K}_{2}(t) \\
\dot{\Delta} \hat{\boldsymbol{f}}(t)
\end{array}\right]=\left[\begin{array}{ccccc}
A_{m} & 0 & 0 & 0 & 0 \\
0 & A & 0 & 0 & B \\
0 & 0 & -\sigma \mathbb{I}_{q \times n} & 0 & 0 \\
0 & 0 & 0 & -\sigma \mathbb{I}_{q \times q} & 0 \\
\gamma B_{m}^{\top} P & -\gamma B_{m}^{\top} P & 0 & 0 & -\sigma \mathbb{I}_{q \times q}
\end{array}\right]\left[\begin{array}{c}
\Delta \boldsymbol{x}^{0}(t) \\
\Delta \boldsymbol{x}(t) \\
\Delta \hat{K}_{1}(t) \\
\Delta \hat{K}_{2}(t) \\
\Delta \hat{\boldsymbol{f}}(t)
\end{array}\right],
$$

where $\Delta$ symbol indicates the perturbation from the equilibrium of the corresponding variables. It is easy 
to see that the stability of the system (79) is determined by the eigenvalues of the matrix

$$
\Xi=\left[\begin{array}{cc}
A & B \\
-\gamma B_{m}^{\top} P & -\sigma \mathbb{I}_{q \times q}
\end{array}\right] .
$$

We notice that in the case of conventional adaptive laws (without the $\hat{\boldsymbol{f}}$-dynamics), instead of the matrix $\Xi$ one gets matrix $A$. That is for the unstable $A$ the trivial equilibrium of the closed-loop system (79) is unstable, hence bursting can take place (see Ref. ${ }^{6}$ for details). With the additional adaptive law for $\hat{\boldsymbol{f}}$, the analysis of the matrix $\Xi$ is not straightforward, but it can be seen that for any $\gamma$ we have $\operatorname{tr}(\Xi)=\operatorname{tr}(A)-q \sigma$. Therefore for small $\sigma$ there can exist $A$ such that $\operatorname{tr}(\Xi)>0$, hence $\Xi$ has eigenvalues with positive real parts. Therefore, the trivial equilibrium can be unstable, which is the source of possible bursting. For the MRAC design, the bursting phenomena for our simulation example is displayed in Figure 14, where we set $\sigma=0.03$ and $\gamma=1$ as in the Ref. ${ }^{6}$ Increasing $\gamma$ reduces the error magnitude, but the phenomena still exists, which can be observed from the Figure 15.
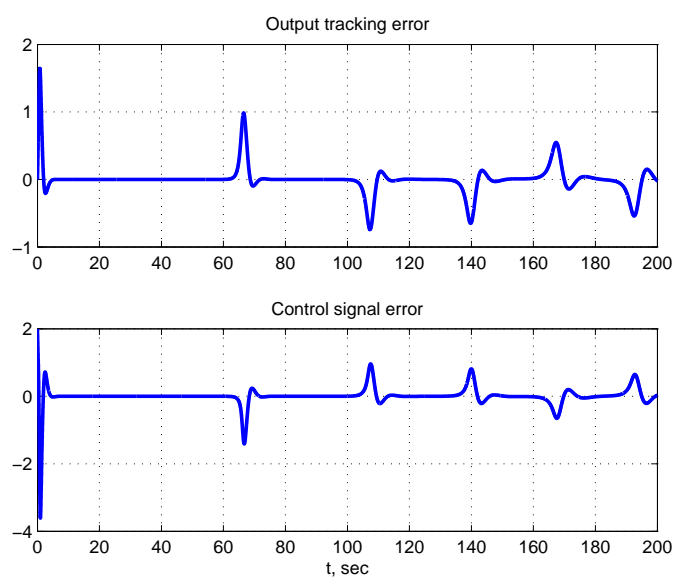

Figure 14. MRAC error signals for $\gamma=1$, bursting.
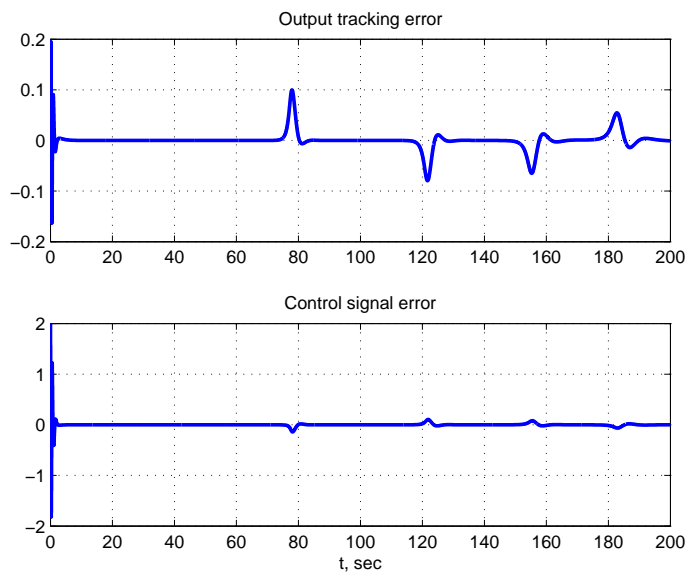

Figure 15. MRAC error signals for $\gamma=100$, bursting. 
For the M-MRAC design the closed-loop error system for $\boldsymbol{r}(t)=0$ and $\boldsymbol{f}(t)=0$ takes the form

$$
\begin{aligned}
\dot{\boldsymbol{x}}_{m}(t) & =A_{m} x_{m}(t)+\lambda\left[\boldsymbol{x}(t)-\boldsymbol{x}_{m}(t)\right] \\
\dot{\boldsymbol{x}}(t) & =A \boldsymbol{x}(t)+B\left[\hat{K}_{1}(t) \boldsymbol{x}(t)+\hat{\boldsymbol{f}}(t)\right] \\
\dot{\hat{K}}_{1}(t) & =-\gamma B_{m}^{\top} P\left[\boldsymbol{x}(t)-\boldsymbol{x}_{m}(t)\right] \boldsymbol{x}^{\top}(t)-\sigma \hat{K}_{1}(t) \\
\dot{\hat{K}}_{2}(t) & =-\sigma \hat{K}_{2}(t) \\
\dot{\hat{\boldsymbol{f}}}(t) & =-\gamma B_{m}^{\top} P\left[\boldsymbol{x}(t)-\boldsymbol{x}_{m}(t)\right]-\sigma \hat{\boldsymbol{f}}(t) .
\end{aligned}
$$

which again has a trivial equilibrium. The linearized equation in this case is written as

$$
\left[\begin{array}{c}
\dot{\Delta \boldsymbol{x}_{m}}(t) \\
\dot{\Delta} \boldsymbol{x}(t) \\
\dot{\Delta \hat{K}_{1}(t)} \\
\dot{\Delta \hat{K}_{2}(t)} \\
\dot{\Delta} \hat{\boldsymbol{f}}(t)
\end{array}\right]=\left[\begin{array}{ccccc}
A_{m}-\lambda \mathbb{I} & \lambda \mathbb{I} & 0 & 0 & 0 \\
0 & A & 0 & 0 & B \\
0 & 0 & -\sigma \mathbb{I}_{q \times n} & 0 & 0 \\
0 & 0 & 0 & -\sigma \mathbb{I}_{q \times q} & 0 \\
\gamma B_{m}^{\top} P & -\gamma B_{m}^{\top} P & 0 & 0 & -\sigma \mathbb{I}_{q \times q}
\end{array}\right]\left[\begin{array}{c}
\Delta \boldsymbol{x}_{m}(t) \\
\Delta \boldsymbol{x}(t) \\
\Delta \hat{K}_{1}(t) \\
\Delta \hat{K}_{2}(t) \\
\Delta \hat{\boldsymbol{f}}(t)
\end{array}\right],
$$

the stability of which is determined by the eigenvalues of the matrix

$$
\Xi_{m}=\left[\begin{array}{ccc}
A_{m}-\lambda \mathbb{I} & \lambda \mathbb{I} & 0 \\
0 & A & B \\
\gamma B_{m}^{\top} P & -\gamma B_{m}^{\top} P & -\sigma \mathbb{I}_{q \times q}
\end{array}\right] .
$$

The stability analysis of the matrix $\Xi_{m}$ is complicated. However, one can notice that unlike the MRAC case, by increasing $\lambda$ one can achieve $\operatorname{tr}(\Xi)=\operatorname{tr}\left(A_{m}-\lambda \mathbb{I}\right)+\operatorname{tr}(A)-q \sigma<0$, no matter how small $\sigma$ is. This enables as to avoid bursting by the proper choice of $\gamma$. For the same simulation example with M-MRAC architecture from Section IV, no bursting can be observed (see Figure 14), when we select $\gamma=100$, which corresponds to $\lambda=17.9322$. In fact, $\Xi_{m}$ is stable for all $\gamma \geq 53$.
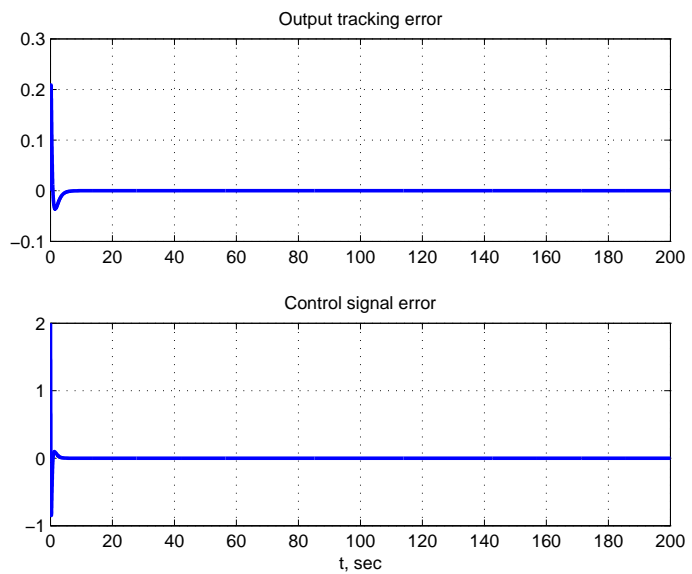

Figure 16. M-MRAC error signals $(\gamma=100)$, no bursting.

\section{Conclusions}

We have presented performance analysis of modified reference model MRAC algorithm with several robust adaptive laws in the presence of bounded disturbances. It is shown that the systems' both input and output tracking errors can be decreased as desired by increasing the adaptation rate when the error feedback gain is selected according to derived rule. This design method prevents high frequency oscillations in the control signal, which are unavoidable in conventional MRAC systems. The performance of M-MRAC is uniform in the sense of the choice of form of robust adaptive laws. Moreover, M-MRAC also prevents bursting of the tracking error, when the external input is not sufficiently rich. 


\section{References}

${ }^{1}$ A. M. Artega and Y. Tang. Adaptive Control of Robots with an Improved Transient performance. IEEE Trans. Autom. Contr., 47(7):1198-1202, 2002.

${ }^{2}$ C. Cao and N. Hovakimyan. Design and Analysis of a Novel $\mathcal{L}_{1}$ Adaptive Control Architecture with Guaranteed Transient Performance. IEEE Trans. Autom. Contr., 53(2):586-591, 2008.

${ }^{3}$ A. Datta and M.-T. Ho. On Modifying Model Reference Adaptive Control Schemes for performance improvement. IEEE Trans. Autom. Contr., 39(9):1977-1980, 1994.

${ }^{4}$ A. Datta and P. Ioannou. Performance Analysis and Improvement in Model Reference Adaptive Control. IEEE Trans. Autom. Contr., 39(12):2370-2387, 1994.

${ }^{5}$ M. French, Cs. Szepesvari, and E. Rogers. Uncertaity, Performance, and Model Dependency in Approximate Adaptive Nonlinear Control. IEEE Trans. Autom. Contr., 45(2):353 - 358, 2000.

${ }^{6}$ L. Hsu and R. R. Costa. Bursting Phenomena in Continuous-Time Adaptive Systems with $\sigma$-Modification. IEEE Trans. Autom. Contr., 32(1):84 - 86, 1987.

${ }^{7}$ P.A. Ioannou and P.V. Kokotovic. Instability analysis and improvement of robustness of adaptive control. Automatica, 20(5):583-594, 1984.

${ }^{8}$ H.K. Khalil. Nonlinear Systems, Third Edition. Prentice Hall, New Jersey, 2002.

${ }^{9}$ M. Krstic, I. Kanellakopoulos, and P. Kokotovic. Nonlinear and Adaptive Control Design. John Wiley \& Sons, New York, 1995.

${ }^{10} \mathrm{~S}$. Morse. Supervisory Control of Families of Linear Set-Point Controllers - Part 1: Exact Matching. IEEE Trans. Autom. Contr., 41(10):1413-1431, 1996.

${ }^{11} \mathrm{~S}$. Morse. Supervisory Control of Families of Linear Set-Point Controllers - Part 1: Robustness. IEEE Trans. Autom. Contr., 42(11):1500-1515, 1997.

${ }^{12}$ K.S. Narendra and A.M. Annaswamy. A New Adaptive Law for Robust Adaptation Without Persistent Excitation. IEEE Trans. Autom. Contr., 32(2):134-145, 1987.

${ }^{13}$ K.S. Narendra and A.M. Annaswamy. Stable Adaptive Control. Prentice Hall, 1989.

${ }^{14}$ B.B Peterson and K.S. Narendra. Bounded Error Adaptative Control. IEEE Trans. Autom. Contr., 27:1161-1168, 1982.

${ }^{15}$ J. B. Pomet and L. Praly. Adaptive Nonlinear Regulation: Estimation from the Lyapunov Equation. IEEE Trans. Autom. Contr., 37(6):729-740, 1992.

${ }^{16}$ C. Samson. Stability Analysis of Adaptively Controlled Systems Subject to Bounded Disturbances. Automatica, 19:81-86, 1983.

${ }^{17}$ V. Stepanyan and K. Krishnakumar. Modified Reference Model MRAC Has Guaranteed Input-Output Performance. The International Journal of Control, Submitted 2010.

${ }^{18}$ V. Stepanyan and K. Krishnakumar. MRAC Revisited: Guaranteed Performance with Reference Model Modification. In Proc. of the American Control Conference, Baltimore, NJ, Accepted 2010.

${ }^{19}$ V. Stepanyan, K. Krishnakumar, and N. Nguyen. Transient Performance and Asymptotic Tracking with Filtering Robust Adaptive Control. In Proc. of the IEEE Aerospace Conference, Big Sky, MT, 2009.

${ }^{20}$ J. Sun. A Modified Model Reference Adaptive Control Schemes for performance improvement. IEEE Trans. Autom. Contr., 38(7):1255-1259, 1993.

${ }^{21} \mathrm{Z}$. Zang and R. Bitmead. Transient bounds for adaptive control systems. In Proceedings of the 30th IEEE Conference on Decision and Control, pages 2724-2729, December 1990.

${ }^{22}$ Y. Zhang and P.A. Ioannou. A New Linear Adaptive Controller: Design, Analysis and Performance. IEEE Trans. Autom. Contr., 45(5):883 - 897, 2000.

${ }^{23}$ K. Zhou, J. Doyle, and K. Glover. Robust and Optimal Control. Prentice Hall, Inc., NJ, 1996. 